\title{
Vertical Tire Forces Estimation of Multi-Axle Trucks Based on an Adaptive Treble Extend Kalman Filter
}

\author{
Buyang Zhang ${ }^{1,2}$, Ting $\mathrm{Xu}^{1 *} \mathbb{0}$, Hong Wang ${ }^{3}$, Yanjun Huang ${ }^{4}$ and Guoying Chen ${ }^{2}$
}

\begin{abstract}
Vertical tire forces are essential for vehicle modelling and dynamic control. However, an evaluation of the vertical tire forces on a multi-axle truck is difficult to accomplish. The current methods require a large amount of experimental data and many sensors owing to the wide variation of the parameters and the over-constraint. To simplify the design process and reduce the demand of the sensors, this paper presents a practical approach to estimating the vertical tire forces of a multi-axle truck for dynamic control. The estimation system is based on a novel vertical force model and a proposed adaptive treble extend Kalman filter (ATEKF). To adapt to the widely varying parameters, a sliding mode update is designed to make the ATEKF adaptive, and together with the use of an initial setting update and a vertical tire force adjustment, the overall system becomes more robust. In particular, the model aims to eliminate the effects of the over-constraint and the uneven weight distribution. The results show that the ATEKF method achieves an excellent performance in a vertical force evaluation, and its performance is better than that of the treble extend Kalman filter.
\end{abstract}

Keywords: Estimation theory, Adaptive treble extend Kalman filter, Vehicle dynamics, Multi-axle truck, Vertical tire force estimation

\section{Introduction}

Vertical tire forces are essential in vehicle modelling and dynamic control. It is not difficult to determine the vertical tire forces for a two-axle vehicle. Two types of methods, i.e., a direct measurement and an estimation, can be used to evaluate the vertical tire forces. The costs of a direct measurement are high, whereas the estimation method requires more sensors to ensure the accuracy of the estimation. A wheel-force transducer used in the direct measurement approach is usually more expensive than that of an ordinary car [1]. A smart tire or intelligent tire equipped with sensors [2] can measure the pressure, rolling speed, vertical load [3, 4], and tire slip angle. However, the smart tire is still in its early development stage

\footnotetext{
*Correspondence: xuting0309@126.com

1 Jihua Laboratory, Foshan 528200, Guangdong, China

Full list of author information is available at the end of the article
}

such that the vertical forces are still estimated based on the data collected through other approaches. In the widely used second approach, the vertical forces are estimated or calculated based on vehicle models using the information regarding the states of the vehicle, suspension, and axles. The sensors necessary for this approach are commonly used in high-class vehicles and are not unacceptably expensive. For example, in Refs. [5-7], the following sensors were used: gyrometers for yaw and roll rates, accelerometers for longitudinal and lateral accelerations, and suspension deflections sensors for suspension deflections.

Furthermore, an evaluation of the vertical tire forces of a multi-axle vehicle is not as easy to achieve as that of a two-axle vehicle. The presence of additional axles means that the vehicle system is over-constrained, resulting in a more complex calculation of the vertical tire forces. A multi-axle vehicle usually carries heavy goods in different 
volumes such that the positional center of gravity (CG) is unknown. Because goods can be partly unloaded and may not be uniformly distributed during an operation, more sensors are required, incurring additional costs, making this approach unfeasible for use in mass-produced vehicles. Moreover, the calculation method for a multi-axle truck is based on the dynamic load transfer ratio, resulting in a poor robustness. Therefore, in this paper, a practical and accurate estimation method for an evaluation of the vertical tire forces of a four-axle truck based on an adaptive treble extend Kalman filter (ATEKF) is proposed. This method requires fewer sensors and experimental data, and a dynamic load transfer ratio is not needed.

Two main types of vehicle model are used in the estimation method. A quarter car model is widely used in active suspension control systems or road condition estimation, and is usually applied to describe the vertical forces of the vehicle body, the suspension, and the wheels [8-11]. This model is always used in two-axle vehicles and requires a large amount of experimental dampness and stiffness data.

A roll dynamic model for vertical forces usually considers all vehicle state parameters, such as the acceleration, roll angle, roll rate, pitch angle, and pitch rate [12-15]. The suspensions of a roll dynamic model can be simplified as a holistic spring-damper system [16] or a springdamper system [12-14]. The main problem for the roll dynamic model is the setting of the parameters, such as the distance between the roll axle and the CG, and the rotational inertia. These parameters are difficult to measure experimentally, particularly for a heavy-duty vehicle for which the goods can be unevenly located, and the parameters vary within a large range. When applied to a multi-axle vehicle, the second approach obviously requires the use of more sensors than for a two-axle vehicle. Owing to the complexity of an over-constraint and the variation of the parameters, the quarter car model and roll dynamic model cannot be simply used to describe the vertical tire forces of a multi-axle vehicle. Therefore, this paper proposes the design of a new practical method for a vertical tire force estimation based on a separated vehicle model and a proposed ATEKF based on an extended Kalman filter (EKF).

The Kalman filter is a popular estimation method with a low computational cost [14-17]. As updated versions of a Kalman filter, an EKF [18-22] and a DEKF [23, 24] have already been proven to be accurate and suitable for estimation in nonlinear vehicle dynamic models. However, for a four-axle truck, the rotational inertia and the CG position may vary within a large range, and the complexity of the multi-axle setup mean that there are more unknown parameters and states to estimate.
Furthermore, the practical use of the Kalman Filter requires a limited number of states and parameters [12, $18,25]$. In this study, to estimate the vertical forces in the case of an uneven location of the goods and to eliminate an over-constraint, the four-axle truck is separated into several parts. Therefore, additional local parameters are necessary for the estimation. The use of a DEKF will only increase the complexity of the model, which will become unsuitable for this particular system. Therefore, to address these problems, in this paper, an ATEKF for multi-axle trucks (a four-axle truck) that can maintain the number of estimated states and parameters at an acceptable level is proposed. To adapt to large parameter variations, a sliding mode update of the ATEKF is specifically designed. Moreover, an initial setting update and a vertical tire force adjustment are also designed to make the overall system more robust and accurate. The vehicle roll angle, vehicle equivalent $C G$ position, equivalent stiffness and dampness, equivalent rotational inertia, equivalent stiffness of the anti-roll bar, and equivalent CG position of each part are unknown and estimated during the process. Based on these states and parameters, the vertical forces are calculated and adjusted. The non-uniform distribution characteristics of the load mass are also considered in the estimation. The initial parameters can be measured simply with fewer experiments.

In this research, an estimation system based on an ATEKF was designed to estimate the vertical tire forces of a multi-axle truck with fewer sensors. The proposed ATEKF needs less primary preparation of the parameters, and the estimation is more accurate than that based on an EKF. Owing to the model improvement, the vertical tire forces with unevenly distributed cargo loading can also be estimated. This research is organized as follows: In Section 2, an improved model is presented for modelling the vertical force on the tires of a fouraxle truck. An estimation system of the vertical force, state, and parameters based on the use of an ATEKF is described in Section 3. The analysis results of this estimation system are this presented in Section 4, along with a comparison between the ATEKF and TEKF. Finally, in Section 5, some concluding remarks are provided and areas of future study are discussed.

\section{Modelling}

To observe wheel forces, the model of a four-axle truck in Ref. [26] was improved in this research. This model divides the truck into three parts, where each part can be regarded as an isolated system, and two virtual forces are used to describe the connection between the parts. Thus, the nonuniform distribution characteristics of the load mass and an over-constraint can be considered as improvements. 
In this truck, the suspensions of the second, third, and fourth axles have the same characteristics. The density of the goods is uniform, and the goods can be loaded with different mass, volume, and position (cannot be loaded only on Part 1); however, the density of the goods is symmetrical with respect to the $\mathrm{x}$-axis of the vehicle. The general CG position is not necessary for the model. In Figures 1 and 2, c. $g_{1}, c . g_{2}$, and c.g $g_{3}$ are the local CG points of each part. The mass of Part 1 consists of the unloaded truck, the first axle and a portion of the load. The values of c.g $g_{2}$ and c.g $g_{3}$ in Parts 2 and 3 are assumed at the same position as those of the unloaded truck. The mass of Part 2 consists of the Part 2 cargo mass and the second axle. The second separation point is located between the second and third axles. The mass of Part 3 is composed of the remaining fraction of the good mass and the other two axles. The virtual or hypothetical internal forces $\left(F_{z a i}=F_{z a l i}, F_{z a r i}\right.$, $i=1,2,3$ ) are given at the separation points. The symbols used in the figures and equations are given in "Nomenclature" (Appendix). The vertical forces can be separated into static vertical forces $\left(F_{z r i 0}, z l i 0\right)$ and extra vertical forces $\left(\Delta F_{z r i, z l i}\right)$ as in Eq. (1). The static vertical forces can be easily obtained (shown in Appendix").

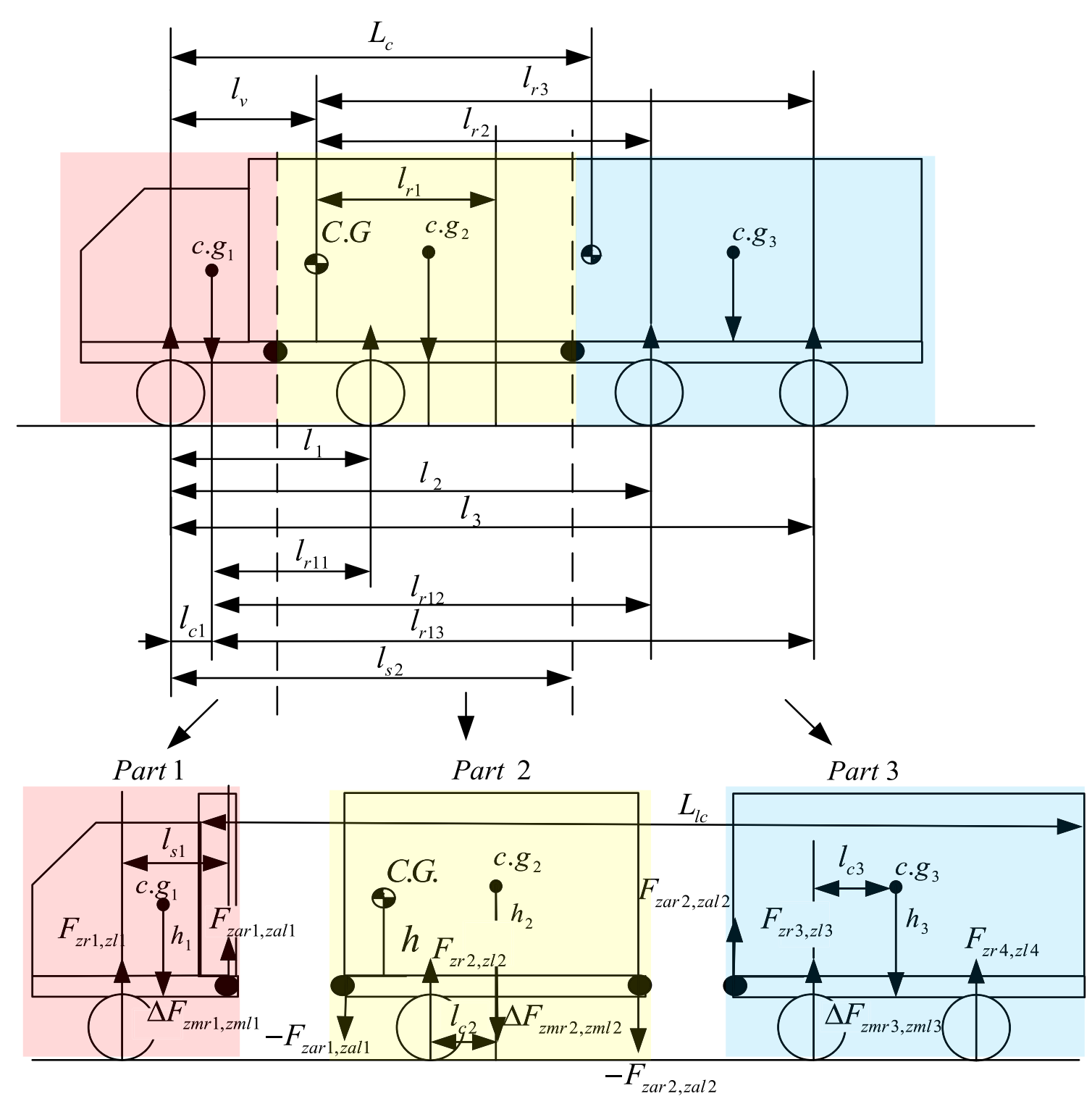

Figure 1 Four-axle truck with three separated parts 


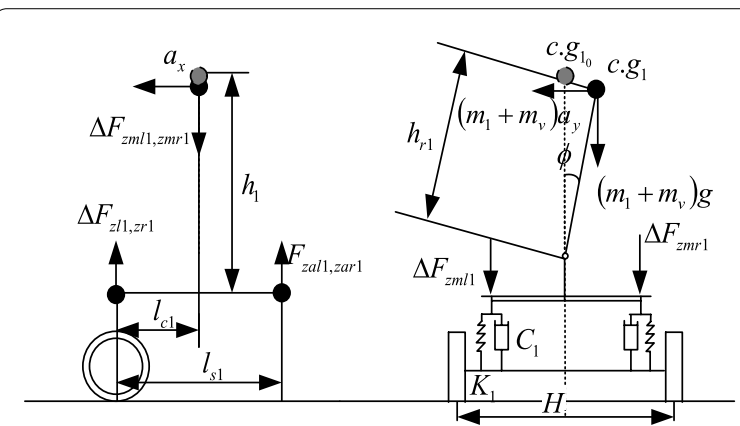

a Part 1

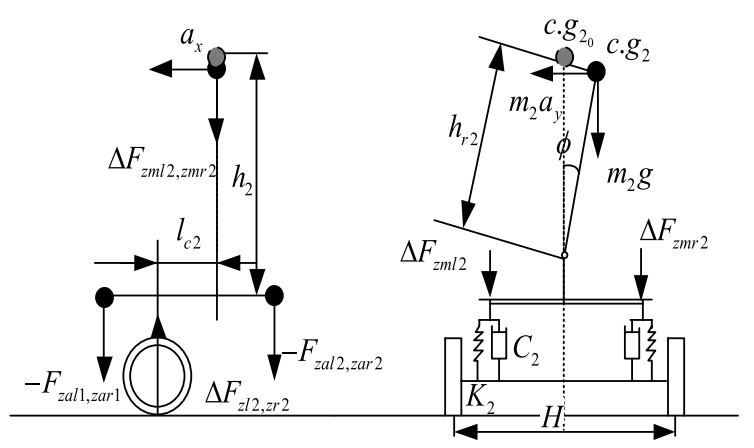

b Part 2

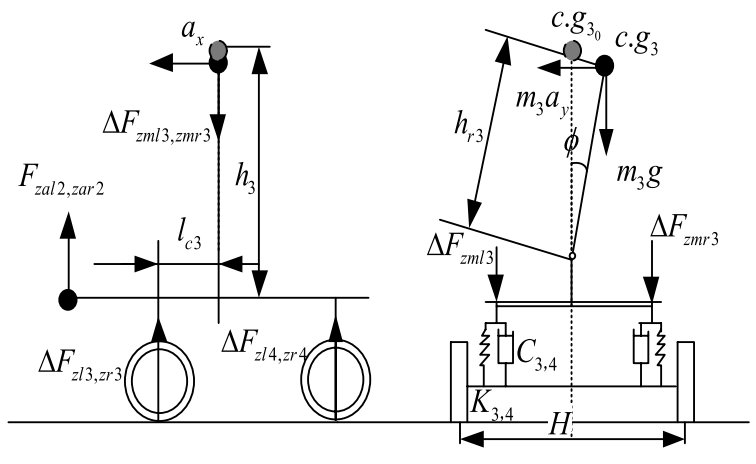

c Part 3

Figure 2 Three separated parts of the truck

$$
\begin{aligned}
& F_{z r i, z l i}=F_{z r i 0, z l i 0}+\Delta F_{z r i, z l i}, \quad i=1,2,3,4, \\
& \left\{\begin{array}{l}
F_{z r i}=F_{z r i 0}+F_{z l i 0}, \quad \text { if } \quad F_{z l i} \leq 0, \\
F_{z l i}=0,
\end{array}\right. \\
& \left\{\begin{array}{l}
F_{z l i}=F_{z r i 0}+F_{z l i 0}, \quad \text { if } \quad F_{z r i} \leq 0 . \\
F_{z r i}=0,
\end{array}\right.
\end{aligned}
$$

To describe the nonuniform distribution characteristics, the loads on each part under different conditions are analyzed and calculated, the details are shown in Table 1. The equivalent distances of $c . g_{1}, c . g_{2}$, and $c . g_{3}$ to the first, second, and third axles with loading $\left(l_{c 1}, l_{c 2}, l_{c 3}\right)$ are given as Eq. (2). For Part 1, a simple model can be established as shown in Figure 2(a). Eqs. (3)-(5) can be obtained from Ref. [26]. Here, $l_{s 1}$ is the distance between the first separation point and the first axle. In addition, $l_{v 1}$ is the distance between point $c . g_{1}$ and the front axle without loading. The rest of the vertical forces can be represented in a similar manner as given by Eqs. (6)-(11), as summarized in Figures 3(b) and 3(c). Different from the models in Ref. [26], in these equations, $m_{1}, m_{2}$, and $m_{3}$ representing the uneven loading masses on each part are from Table 1.

$$
\left\{\begin{array}{l}
l_{c 1}=\frac{m_{1}}{\left(m_{1}+m_{v}\right)}\left(l_{s 1}-\frac{L_{1}}{2}\right), \quad l_{s 1}=l_{v 1}+\frac{l_{r 11}}{2}, \\
l_{c 2}=\frac{\left(\frac{l_{2}-l_{1}}{2}+l_{1}\right)-l_{s 1}}{2}+l_{s 1}-l_{1}, \\
l_{c 3}=\frac{l_{3}-\left(\frac{l_{2}-l_{1}}{2}+l_{1}\right)}{2}+\left(\frac{l_{2}-l_{1}}{2}+l_{1}\right)-l_{2} .
\end{array}\right.
$$

\section{Functions for Part 1}

$$
\begin{gathered}
\Delta F_{z r 1, z l 1}=\Delta F_{z m r 1, z m l 1}-F_{z a r 1, z a l 1} \pm \frac{K_{b 1} \phi}{H} \\
\begin{aligned}
\Delta F_{z m r 1, z m l 1}= & \pm \frac{\left(m_{\nu} a_{y} h_{1}+m_{1} a_{y} h_{1}\right)}{H} \cos (\phi) \\
& \pm \frac{\left(K_{1} \phi+C_{1} \dot{\phi}\right)}{H} \\
& \pm \frac{\left(m_{\nu} g h_{r 1}+m_{1} g h_{r 1}\right) \sin \phi}{H} \\
F_{z a r 1, z a l 1}= & \frac{\Delta F_{z m r 1, z m l 1} l_{c 1}+m_{\nu} a_{x} h_{1} / 2+m_{1} a_{x} h_{c} / 2}{l_{s 1}} .
\end{aligned}
\end{gathered}
$$

\section{Functions for Part 2}

$$
\begin{gathered}
\Delta F_{z r 2, z l 2}=-\left(F_{z a r 2, z a l 2}+F_{z a r 1, z a l 1}-\Delta F_{z m r 2, z m l 2}\right) \pm \frac{K_{b 2} \phi}{H}, \\
\Delta F_{z m r 2, z m l 2}= \pm \frac{m_{2} a_{y} h_{2}}{H} \cos (\phi) \pm \frac{\left(K_{2} \phi+C_{2} \dot{\phi}\right)}{H} \\
\pm \frac{m_{2} g h_{r 2} \sin \phi}{H},
\end{gathered}
$$


Table 1 Parameter design based on different loaded positions $\left(L_{i}=\right.$ length of goods in Part $1, m_{i}=$ cargo mass in Part 1$)$

$$
\begin{aligned}
& \text { Parameters for Part } 1 \\
& \left\{\begin{array}{l}
L_{1}=\left(I_{v 1}+\frac{I_{r 11}}{2}\right)-\left(L_{c}-\frac{L_{c}}{2}\right), \\
m_{1}=L_{1} \frac{m_{c}}{L_{c}},
\end{array}\right. \\
& \left\{\begin{array}{r}
L_{1}=0, \\
m_{1}=0,
\end{array} \text { if }\left(I_{v 1}+\frac{I_{r 11}}{2}\right)-\left(L_{c}-\frac{L_{c}}{2}\right)<0 .\right. \\
& \text { Parameters for Parts } 2 \text { and } 3 \\
& \left\{\begin{array}{l}
L_{2}=L_{k}-L_{1}, \\
L_{3}=0, \\
m_{2}=m_{c}-m_{1}, \quad \text { if } L_{c}-\frac{L_{k}}{2}<\frac{l_{2}-l_{1}}{2}+I_{1} \text { and } L_{c}+\frac{L_{c}}{2} \leq \frac{l_{2}-I_{1}}{2}+I_{1} . \\
m_{3}=0,
\end{array}\right. \\
& \begin{cases}L_{2}=\left(\frac{I_{2}-I_{1}}{2}+I_{1}\right)-\left(L_{c}-\frac{L_{C}}{2}\right)-L_{1}, & \\
L_{3}=\left(L_{c}+\frac{L_{c}}{2}\right)-\left(\frac{I_{2}-I_{1}}{2}+I_{1}\right), & \text { if } L_{c}-\frac{L_{c}}{2}<\frac{I_{2}-I_{1}}{2}+I_{1} \\
m_{2}=\frac{m_{c}}{L_{c}}\left[\left(\frac{I_{2}-I_{1}}{2}+I_{1}\right)-\left(L_{c}-\frac{L_{c}}{2}\right)\right]-m_{1}, L_{c}+\frac{L_{c}}{2}>\frac{I_{2}-I_{1}}{2}+I_{1} . \\
m_{3}=\frac{m_{c}}{L_{c}}\left[\left(L_{c}+\frac{L_{c}}{2}\right)-\left(\frac{I_{2}-I_{1}}{2}+I_{1}\right)\right],\end{cases} \\
& \left\{\begin{aligned}
L_{2} & =0 \\
L_{3} & =L_{c,} \\
m_{2} & =0 \\
m_{3} & =m_{c}
\end{aligned} \text { if } L_{c}-\frac{L_{c}}{2} \geq \frac{l_{2}-l_{1}}{2}+I_{1} .\right.
\end{aligned}
$$

$$
\begin{aligned}
F_{z a r 2, z a l 2}= & \frac{-\left(F_{z a r 1, z a l 1} l_{r 11} / 2+m_{2} a_{x} h_{2} / 2\right)}{\left(l_{r 12}-l_{r 11}\right) / 2} \\
& +\frac{\Delta F_{z m r 2, z m l 2} l_{c 2}}{\left(l_{r 12}-l_{r 11}\right) / 2} .
\end{aligned}
$$

\section{Functions for Part 3}

$$
\begin{aligned}
\Delta F_{z m r 3, z m l 3}= & \pm \frac{m_{3} a_{y} h_{3}}{H} \cos (\phi) \pm \frac{\left(K_{3} \phi+C_{3} \dot{\phi}\right)}{H} \\
& \pm \frac{m_{3} g h_{r 3} \sin \phi}{H}
\end{aligned}
$$

should be used for estimation. This research proposes an estimation system shown in Figure 3. This estimation system has three subsystems: one vertical force on the second axle achievement module, an ATEKF estimation module, and a vertical force achievement and adjustment module. In this estimation, only one wheel on the second axle is assumed to have vertical acceleration sensors implanted, and the vertical tire forces can be obtained using the $1 / 8$ vehicle model based on the sensor data [27]. Because this method has already been used commercially, during the estimation, this vertical force $\left(F_{z l 2}\right)$ is considered to be known as the input of the ATEKF. The

$$
\Delta F_{z r 3, z l 3}=\frac{\left(-F_{z a r 2, z a l 2}\left(\frac{\left(l_{r 12}-l_{r 11}\right)}{2}+\left(l_{r 13}-l_{r 12}\right)\right)\right)}{l_{r 13}-l_{r 12}}+\frac{\frac{m_{3} a_{x} h_{3}}{2}+\Delta F_{z m r 3, z m l 3}\left(l_{r 13}-l_{r 12}-l_{c 3}\right)}{l_{r 13}-l_{r 12}} \pm \frac{K_{b 3} \phi}{H},
$$

$$
\Delta F_{z r 4, z l 4}=\Delta F_{z r 3, z l 3}+F_{z a r 2, z a l 2}+\Delta F_{z m r 3, z m l 3} \pm \frac{K_{b 4} \phi}{H} .
$$

\section{Estimation System Based on ATEKF}

The vertical tire force model in Section 2 is nonlinear, because $a_{y}$ and $K_{b i}(i=2,3,4)$ are always changing with the driver operations. Therefore, a nonlinear method parameters of Part 1 are also considered to be known because they are close to the parameters for the unloaded truck. The proposed estimation system has only three sensors: two accelerometers (for vertical accelerations of the wheels), and one inertial navigation sensor for the roll angle rate, longitudinal acceleration, and lateral acceleration of the vehicle body (located at the driver position). This is less than the number of sensors used in the $1 / 8$ 


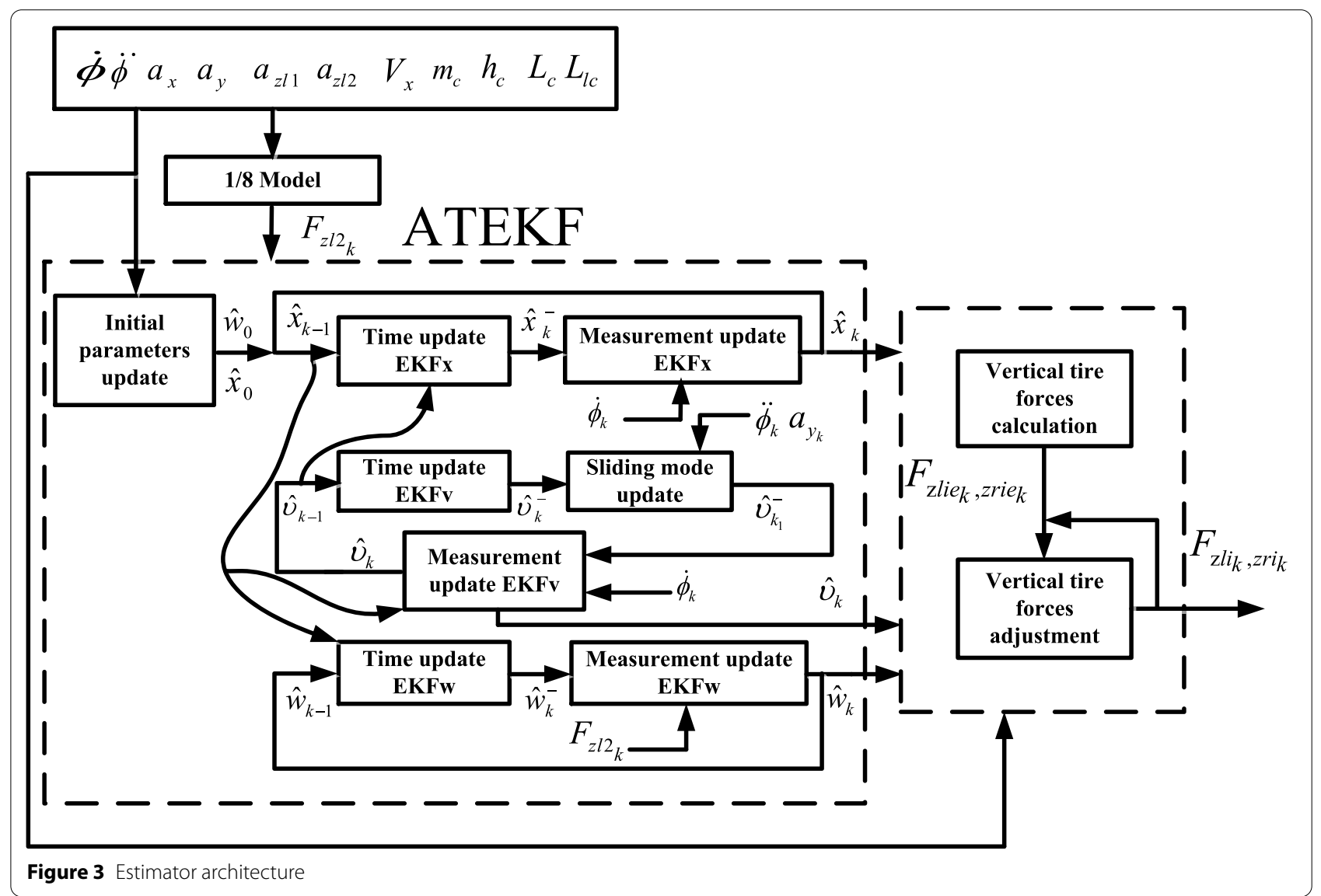

models (but at least 8). The vehicle roll angle, the vehicle equivalent CG position, the equivalent stiffness and dampness of the vehicle, the equivalent rotational inertia, the equivalent stiffness of anti-roll bar, and the equivalent CG position of each part are unknown and are estimated during the process through the ATEKF estimation module. Other parameters used in the estimation can be directly measured. The value of $\ddot{\phi}$ is derived from the roll angle rate $\dot{\phi}$, which is adopted for the sliding mode update during the estimation process. With the estimated parameters and states, the vertical tire forces are finally calculated and adjusted by the vertical force achievement and adjustment module.

\subsection{ATEKF Estimation Module}

The ATEKF estimation module is the most important part of the entire estimation system. The proposed ATEKF is improved from the EKF, and has an initial parameter update and an adaptive function based on the slide mode algorithm. These two functions can decrease the previous preparation and increase the estimation efficiency. The ATEKF algorithm estimates the states and identifies the parameters involved in the operation (the vehicle equivalent $C G$ position, the equivalent stiffness and dampness of the vehicle, and the equivalent rotational inertia) based on a roll model. In addition, the other parameters (the equivalent stiffness of the antiroll bar, and the equivalent CG position of each part) are identified using the vertical force model in Section 2. The details of the ATEKF estimation module are shown in Figure 3.

The nonlinear roll model is described through Eq. (12). The state-space model in the estimation can be described using Eq. (13). In the equation, $\tau_{k}$ is the process noise, and $\varepsilon_{k}$ and $\varsigma_{k}$ are the observation noise. In addition, $v$ and $w$ are the parameters of the dynamics model (Eq. (12)) and Part 2 (Eqs. (6)-(8)). The other symbols are described in "Nomenclature".

$$
\begin{aligned}
& J_{x} \ddot{\phi}=h \cos (\phi) m a_{y}-K \phi-C \dot{\phi}, \\
& \left\{\begin{aligned}
x_{k+1} & =F_{x}\left(x_{k}, a_{y_{k}}, v_{k}\right)+\tau_{k}, \\
y_{1_{k}} & =\left[\begin{array}{ll}
1 & 0
\end{array}\right] x_{k}+\varepsilon_{k} \\
y_{2_{k}} & =F_{z 2}\left(x_{k}, w_{k}, a_{y_{k}}, a_{x_{k}}\right)+\varsigma_{k} \\
& =F_{z l 2_{0}}+\Delta F_{z l 2}\left(x_{k}, w_{k}, a_{y_{k}}, a_{x_{k}}\right)+\varsigma_{k},
\end{aligned}\right.
\end{aligned}
$$

where 


$$
\begin{aligned}
& x=\left[\begin{array}{l}
\dot{\phi} \phi
\end{array}\right]^{\mathrm{T}}, v=\left[\begin{array}{lll}
a & b & c
\end{array}\right]^{\mathrm{T}}, w=\left[\begin{array}{lll}
h_{2} & h_{r 2} & K_{b 2}
\end{array}\right]^{\mathrm{T}}, \\
& a=K_{a} h / J_{x}, b=K / J_{x}, c=C / J_{x} \text {. }
\end{aligned}
$$

Here, $K_{a}$ is the gain for unifying the orders of magnitude. The initial settings of ATEKF are shown as Eq. (14), and the others are listed in "Nomenclature".

$$
\left\{\begin{array}{l}
v_{0}=\left[\begin{array}{lll}
a_{0} & b_{0} & c_{0}
\end{array}\right]^{\mathrm{T}}, \\
x_{0}=\left[\begin{array}{ll}
0 & 0
\end{array}\right]^{\mathrm{T}}, \\
w_{0}=\left[\begin{array}{lll}
1.2 & 0.1 & 1.4
\end{array}\right]^{\mathrm{T}} .
\end{array}\right.
$$

\subsubsection{Initial Parameter Update}

To avoid large errors and improve the estimation speed, the initially set value of $a$ is updated for different cargo loads and sizes. In addition, $J_{x}$ has a strong influence on the initial values, and therefore it will be updated for different cargo weights. Eq. (15) is a rough model for the truck rotary inertia about the $x$-axis based on the parallel axis theorem. The value of parameter $d$ (height of the cargo) also has a large range of variation, and therefore the initial value of $a$ is described through Eq. (16):

$$
\begin{aligned}
& J_{x 0}=\left[J_{x c}+m_{c}\left(\frac{d}{2}+h_{b}\right)^{2}+J_{x t}+m_{v} h_{d}^{2}\right], \\
& a_{0}=K_{J x 1}\left(\frac{m_{c} d}{2 m_{s} J_{x 0}}\right)+K_{J x 2},\left\{\begin{array}{l}
m_{s}=m_{c}+m_{v}, \\
J_{x c}=\frac{1}{12} m_{c}\left(H_{c}^{2}+d^{2}\right),
\end{array}\right.
\end{aligned}
$$

where $K_{J x 1}$ and $K_{J x 2}$ are gains, $J_{x c}$ is the rotational inertia of cargo about the $x$-axis, $J_{x t}$ is the unloaded truck rotational inertia about the $x$-axis, and $h_{d}$ is the distance between a hypothetical roll axle and vehicle roll axle.

\subsubsection{ATEKF}

The ATEKF algorithm consists of three EKF algorithms and one sliding mode update. The time-update equations for the parameters are as follows:

$\hat{v}_{k}^{-}=\hat{v}_{k-1}^{-}, \quad \hat{w}_{k}^{-}=\hat{w}_{k-1}^{-}, \quad P_{v_{k}}^{-}=\frac{P_{v_{k-1}}}{\lambda_{1}}, \quad P_{w_{k}}^{-}=\frac{P_{w_{k-1}}}{\lambda_{2}}$, $\lambda_{1}=0.1, \lambda_{2}=0.1$.

The equations for the states filter are

$$
\left\{\begin{array}{l}
\hat{x}_{k}^{-}=F_{x}\left(\hat{x}_{k-1}^{-}, a_{y_{k}}, \hat{v}_{s_{k}}^{-}\right), \\
P_{x_{k}}^{-}=A_{k-1} P_{x_{k-1}} A_{k-1}^{T}+R^{\tau} .
\end{array}\right.
$$

In addition, the measurement-update equations for the filter states are as follows:

$$
\begin{aligned}
& K_{k}^{x}=P_{x_{k}}^{-} A_{l 1}^{T}\left(A_{l 1} P_{x_{k}}^{-} A_{l 1}^{T}+R^{n}\right)^{-1}, \\
& \left\{\begin{array}{l}
\hat{x}_{k}=\hat{x}_{k}^{-}+K_{k}^{x}\left(\dot{\phi}_{k}-\hat{\dot{\phi}}_{k}^{-}\right), \\
P_{x_{k}}=\left(I-K_{k}^{x} A_{l 1}\right) P_{x_{k}}^{-} .
\end{array}\right.
\end{aligned}
$$

The update equations for the parameters in the roll dynamic model are as follows:

$$
\begin{aligned}
& K_{k}^{v}=P_{v_{k}}^{-} A_{v_{k}}^{T}\left(A_{v_{k}} P_{v_{k}}^{-} A_{v_{k}}^{T}+R^{e}\right)^{-1}, \\
& \hat{v}_{k 1}^{-}=\hat{v}_{k}^{-}-K_{e s} s a t\left(\frac{\hat{\ddot{\phi}}_{k}-\ddot{\phi}_{k}}{m a_{y_{k}}}\right), \\
& \left\{\begin{array}{l}
\hat{v}_{k}=\hat{v}_{k_{1}}^{-}+K_{k}^{v}\left(\dot{\phi}_{k}-F_{x}\left(\hat{x}_{k}^{-}, a_{y_{k}}, \hat{v}_{k}^{-}\right)\right), \\
P_{v_{k}}=\left(I-K_{k}^{v} A_{v_{k}}\right) P_{v_{k}}^{-} .
\end{array}\right.
\end{aligned}
$$

Finally, the update equations for Part 2 are

$$
\begin{aligned}
& K_{k}^{w}=P_{w_{k}}^{-} A_{w l 2_{k}}^{T}\left(A_{w l 2_{k}} P_{w_{k}}^{-} A_{w l 2_{k}}^{T}+R^{f}\right)^{-1}, \\
& \left\{\begin{array}{c}
\hat{w}_{k}=\hat{w}_{k}^{-}+K_{k}^{w}\left(F_{z l 2_{k}}-F_{z 2}\left(\hat{x}_{k}^{-}, \hat{w}_{k}^{-}, a_{y_{k}}, a_{x_{k}}\right)\right), \\
P_{w_{k}}=\left(1-K_{k}^{w} A_{w l 2_{k}}\right) P_{w_{k}}^{-},
\end{array}\right.
\end{aligned}
$$

$$
\begin{aligned}
& A_{k-1}=\left.\frac{\partial F_{x}\left(x, \hat{v}_{k}^{-}\right)}{\partial x}\right|_{\hat{x}_{k-1}}, A_{l 1}=\left[\begin{array}{ll}
1 & 0
\end{array}\right] \\
& A_{v_{k}}=\left.\frac{\partial F_{x}\left(\hat{x}_{k}, v\right)}{\partial v}\right|_{v=\hat{v}_{k}^{-}} A_{w l 2_{k}}=\left.\frac{\partial F_{z 2}\left(\hat{x}_{k^{-}}, w, a_{y_{k}}, a_{x_{k}}\right)}{\partial w}\right|_{w=\hat{w}_{k}^{-}} .
\end{aligned}
$$

The sliding mode update described by Eq. (21) in the ATEKF for the parameters of the roll dynamic model is proposed to decrease the estimation errors. Its operational principle is shown in Figure 4. Prior to the transformation from $\hat{x}_{k}^{-}$to $y\left(\hat{x}_{k}^{-}\right)$, a sliding surface is designed for converting $\hat{x}_{k}^{-}$into $\hat{x}_{k_{1}}^{-}$, which is closer to the real value $x_{\text {real }}$. The sliding mode update transforms $\hat{x}_{k_{1}}^{-}$into $y\left(\hat{x}_{k_{1}}^{-}\right)$. After the measurement update, the new $\hat{x}_{k}$ will be closer to the real value. Therefore, together with the initial parameter update, this sliding mode update leads to a faster estimation convergence. Because $a$ is more influential than $b$ and $c, b$ and $c$ are considered to be close to the real values. 


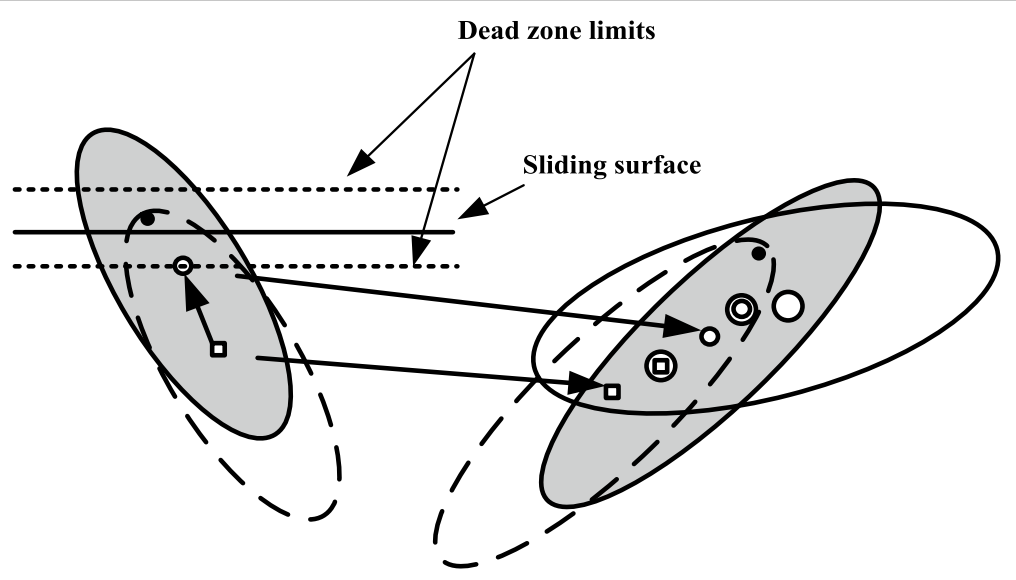

- $x_{\text {real }} y\left(x_{\text {real }}\right)$

○ $\hat{x}_{k_{1}}^{-} y\left(\hat{x}_{k_{1}}^{-}\right)$

口 $\hat{x}_{k}^{-} y\left(\hat{x}_{k}^{-}\right)$

(a) $y\left(\hat{x}_{k_{1}}\right)$

O $z_{\text {measured }}$

(?) $y\left(\hat{x}_{k}\right)$

Figure 4 Sliding mode update subsystem

$$
\begin{aligned}
& \hat{\ddot{\phi}}_{k}-\ddot{\phi}_{k}=\left[\hat{a_{k}^{-}} \cos \left(\hat{\phi}_{k}\right) m a_{y_{k}}-\hat{b}_{k}^{-} \hat{\phi}_{k}-\hat{c}_{k}^{-} \hat{\dot{\phi}}_{k}\right] \cdots \\
& -\left[a_{r} \cos (\phi) m a_{y}-b_{r} \phi_{k}-c_{r} \dot{\phi}_{k}\right] \\
& \approx\left(\hat{a}_{k} m a_{y_{k}}-a_{r} m a_{y_{k}}\right) / K_{a} \text {, } \\
& K_{a}\left(\frac{\hat{\ddot{\phi}}_{k}-\ddot{\phi}_{k}}{m a_{y_{k}}}\right)=\hat{a}_{k}-a_{r}, \\
& S_{k}=C\left(\hat{v}_{k}-v_{r}\right) \\
& =\left[\begin{array}{lll}
1 & 0 & 0
\end{array}\right]\left(\hat{v}_{k}-v_{r}\right)=\left(\frac{\hat{\ddot{\phi}}_{k}-\ddot{\phi}_{k}}{m a_{y_{k}}}\right), \\
& \hat{a}_{k_{1}}^{-}=\hat{a}_{k}^{-}-k_{e s} s a t\left(\frac{\hat{\ddot{\phi}}_{k}-\ddot{\phi}_{k}}{m a_{y_{k}}}\right) \text {, } \\
& \operatorname{sat}\left(\frac{\hat{\ddot{\phi}}_{k}-\ddot{\phi}_{k}}{m a_{y_{k}}}\right)=\left\{\begin{array}{l}
\operatorname{sign}\left(\frac{\hat{\ddot{\phi}}_{k}-\ddot{\phi}_{k}}{m a_{y_{k}}}\right),\left|\frac{\hat{\ddot{\phi}}_{k}-\ddot{\phi}_{k}}{m a_{y_{k}}}\right|>\Im, \\
0,\left|\frac{\hat{\ddot{\phi}}_{k}-\ddot{\phi}_{k}}{m a_{y_{k}}}\right| \leq \Im .
\end{array}\right.
\end{aligned}
$$

Equation (25) can be derived from Eq. (12). Assuming that the real value of $a$ is $a_{r}, \ddot{\phi}_{k}$ is obtained from the measured signal $\dot{\phi}$. A simple relationship is shown in Eq. (26). The sliding mode switching function is shown in Eq. (27), and the sliding mode update function is shown in Eqs. (28) and (29). Based on Eq. (26), $a_{r}<\hat{a}_{k}$ means that $\hat{a}_{k}$ must be decreased, and $a_{r}>\hat{a}_{k}$ indicates that $\hat{a}_{k}$ must be increased. In the equations, $k_{e s}=0.0008\left(0<k_{e s}<1\right)$, $2 \leq a_{r} \leq 6$, and $\Im=0.00001$.

\subsection{Vertical Tire Force Achievement and Adjustment Module}

Along with the lateral acceleration and the longitudinal acceleration from the sensors or models, all vertical forces can be calculated based on the model from Section 2 with the results of the estimation. The vertical tire forces from the calculation can also accumulate errors from the estimation. This is particularly true for the parameters of Part 3, which are simply transferred from the estimation results. Therefore, an adjustment based on the lateral-load transfer ratio (LTR) is proposed for Part 3 to decrease the error accumulation.

The LTR has already been proven to be effective and has been widely used in vehicle dynamic control systems and rollover warning systems [28, 29], and can provide the global roll movement state of the vehicle. The LTR of Part 3 is given by Eq. (30), where $k_{l t r 3, l t r 4}$ are the factors of a rear magnification. Based on the vertical forces model of Part 3, Eq. (32) can be obtained from Eq. (30). The values of $A_{l t r 3, l t r 4}$ and $B_{l t r 3, l t r 4}$ are defined through Eq. (33):

$$
\begin{aligned}
L T R_{3,4}= & \frac{F_{z r 3, z r 4}-F_{z l 3, z l 4}}{F_{z r 3, z r 4}+F_{z l 3, z l 4}}=k_{l t r 3, l t r 4} \frac{2 a_{y} h_{3}}{g H}, \\
h_{3}=h_{2}+ & \frac{\left(\frac{a}{K_{a}} J_{x 0}-h_{2}\right) m_{3}}{\left(m_{2}+k_{h}\right)}, 0.6 \leq h_{3} \leq \frac{d}{2}+h_{b}, \\
2 \frac{K_{b 3 . b 4} \phi}{H} & =k_{l t r 3, l t r 4} \frac{2 a_{y} h_{3}}{g H}\left(F_{z r 3, z r 4}+F_{z l 3, z l 4}\right) \\
& +\left(F_{z l 3, z l 4}-F_{z r 3, z r 4}\right),
\end{aligned}
$$


Table 2 Rules of the adjustments

$$
\begin{aligned}
& \text { Rule } 1 \\
& \text { The 3rd axle } \\
& \operatorname{sat}\left(A_{l t r 3_{k}}-B_{l t r 3_{k}}\right)= \begin{cases}0, \quad i f\left|A_{l t r 3_{k}}-B_{l t r 3_{k}}\right|<K_{l t r 3} \\
\operatorname{sign}\left(A_{l t r 3_{k}}-B_{l t r 3_{k}}\right), \quad \text { else. }\end{cases} \\
& \operatorname{sat}\left(A_{l t r 4_{k}}-B_{l t r 4_{k}}\right)= \begin{cases}0, & \text { if }\left|A_{l t r} 4_{k}-B_{l t r 4_{k}}\right|<K_{l t r 4_{1}} \\
\operatorname{sign}\left(A_{l t r 4_{k}}-B_{l t r} 4_{k}\right), & \text { else. }\end{cases} \\
& \text { The 3rd axle } \quad \operatorname{sat}_{l}\left(\phi_{k}\right)=\left\{\begin{array}{l}
\operatorname{sign}\left(\phi_{k}\right), \quad \text { if } \phi_{k}<0, \\
0, \quad \text { else. }
\end{array}\right. \\
& \operatorname{sat}_{r}\left(\phi_{k}\right)=\left\{\begin{array}{l}
\operatorname{sign}\left(\phi_{k}\right), \\
0, \quad \text { else. }
\end{array}\right. \\
& \text { The 3rd axle } \quad N_{3 m a_{y}}= \begin{cases}0, & \text { if }\left|m a_{y_{k}}\right|>K_{1 m a_{y}} \\
1, & \text { else. }\end{cases} \\
& \text { The 4th axle } \quad N_{4 m a_{y}}= \begin{cases}0, & \text { if }\left|m a_{y_{k}}\right|<K_{2 m a_{y}} \text { or }\left|m a_{y_{k}}\right|>K_{3 m a_{y}} \\
\operatorname{sign}\left(m a_{y_{k}}\right), & \text { else. }\end{cases} \\
& \left\{\begin{aligned}
A_{l t r 3, l t r 4_{k}} & =2 \frac{K_{b 3 . b 4} \phi_{k}}{H}, \\
B_{l t r 3, l t r 4_{k}} & =k_{l t r 3, l t r 4_{k}} \frac{2 a_{y_{k}} h_{3}}{g H}\left(F_{z r 3, z r 4_{k-1}}+F_{z l 3, z l 4_{k-1}}\right) \\
& +\left(F_{z l 3, z l 4_{k-1}}-F_{z r 3, z r 4_{k-1}}\right) .
\end{aligned}\right.
\end{aligned}
$$

Based on the experimental study, three main rules can be obtained for the vertical force adjustment. First, $A_{\text {ltr3,ltr } 4}-B_{\text {ltr3,ltr4 }}>$ threshold means that the forces generated by the anti-roll bar are too large and must be decreased. This is in contrast with the case above when $A_{\text {ltr } 3, \text { tr } 4}-B_{\text {ltr } 3 \text { ltr } 4}<-$ threshold. Second, the value of $\phi_{k}$ has an influence on the directions of the forces generated by the anti-roll bar. Third, if one side of the suspension cannot be compressed or stretched any longer, the anti-roll bar will be beyond its threshold. These three criteria specifying the adjustment rules are simplified and concluded, as shown in Table 2. The parameters in the table are described in "Nomenclature" (provided in "Appendix").

Based on the adjustment rules in Table 2, the gains and vertical tire forces are finally presented in Eqs. (34) and (35) $\left(K_{n}=0.8, N_{3 l, 3 r_{0}}=0.01, N_{4 l, 4 r_{0}}=0.01\right)$. During the

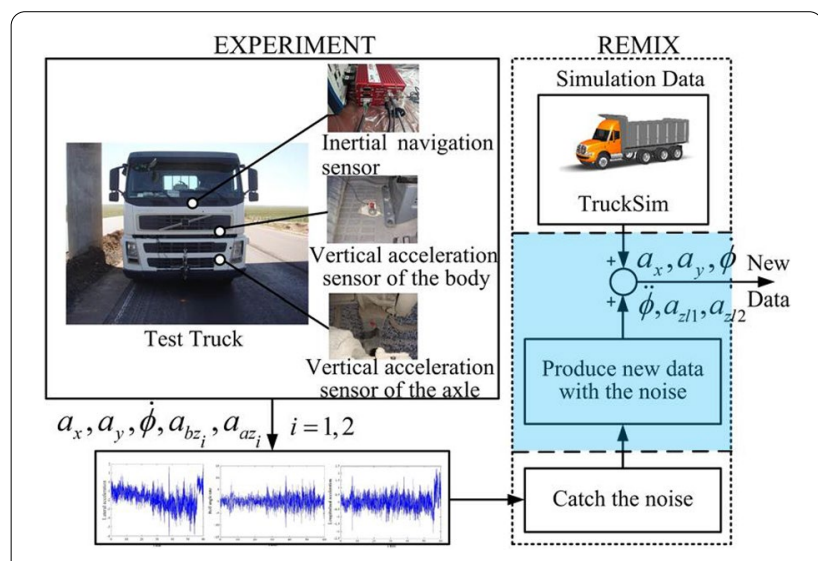

Figure 5 Experiments for the real test noise and data remix

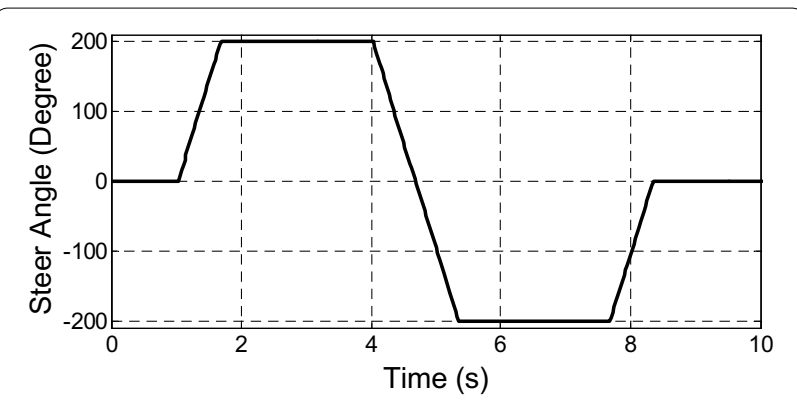

Figure 6 Step steer input

adjustment, $F_{z r 3_{k}, z l 3_{k}}$ and $F_{z r 4_{k}, z l 4_{k}}$ will be sent back for adjustment to the calculations of $A_{l t r 3, l t r 4}$ and $B_{l t r 3, l t r 4}$ in the next step. The values of $F_{z r 3 e_{k}, z l 3 e_{k}}$ and $F_{z r 4 e_{k}, z l 4 e_{k}}$ are directly calculated using the vertical force model.

$$
\begin{aligned}
& \left\{\begin{aligned}
N_{3 l, 3 r_{k+1}} & =N_{3 l, 3 r_{k}} \cdots \\
& -N_{3 m a_{y}} K_{n} \operatorname{sat} l_{l, r}\left(\phi_{k}\right) \operatorname{sat}\left(A_{l t r 3_{k}}-B_{l t r 3_{k}}\right), \\
N_{4 l, 4 r_{k+1}} & =N_{4 l, 4 r_{k}} \cdots \\
& -N_{4 m a_{y}} K_{n} \operatorname{sign}\left(\phi_{k}\right) \operatorname{sat}\left(A_{l t r 4_{k}}-B_{l t r 4_{k}}\right),
\end{aligned}\right. \\
& \left\{\begin{array}{l}
F_{z l 3_{k}, z r 3_{k}}=F_{z l 3 e_{k}, z r 3 e_{k}} \pm N_{3 l, 3 r_{k}} \frac{K_{b 2} \phi}{H} \\
F_{z l 4_{k}, z r 4_{k}}=F_{z l 4 e_{k}, z r 4 e_{k}} \pm N_{4 l, 4 r_{k}} \frac{K_{b 2} \phi}{H}
\end{array}\right.
\end{aligned}
$$

Table 3 Conditions

\begin{tabular}{lllllll}
\hline Condition & $\boldsymbol{L}_{\boldsymbol{c}}(\mathbf{m})$ & $\boldsymbol{L}_{\boldsymbol{c}}(\mathbf{m})$ & $\boldsymbol{d}(\mathbf{m})$ & $\boldsymbol{m}_{\boldsymbol{c}}(\mathbf{k g})$ & Velocity $(\mathbf{k m} / \mathbf{h})$ & Inputs \\
\hline 1 & 7 & 6 & 0.45 & 5000 & 80 & Step steer \\
2 & 3 & 7 & 1.8 & 5000 & 60 & Double lane change \\
3 & 3 & 7 & 1.8 & 20000 & 60 & Step steer \\
4 & 7 & 6 & 1.5 & 10000 & 80 & Step steer with brake \\
\hline
\end{tabular}



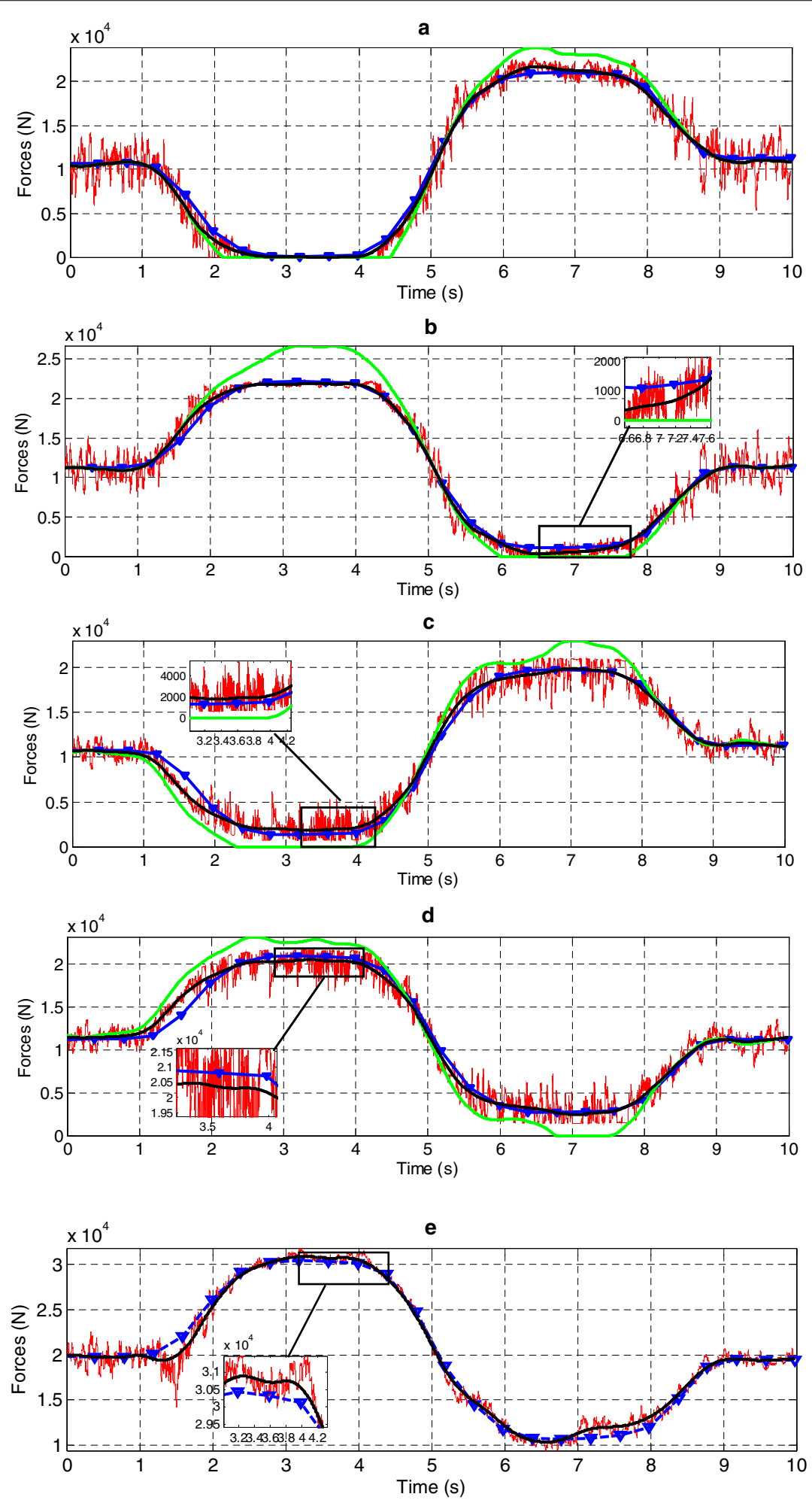

Figure 7 Comparisons between Trucksim and estimation under Condition 1 


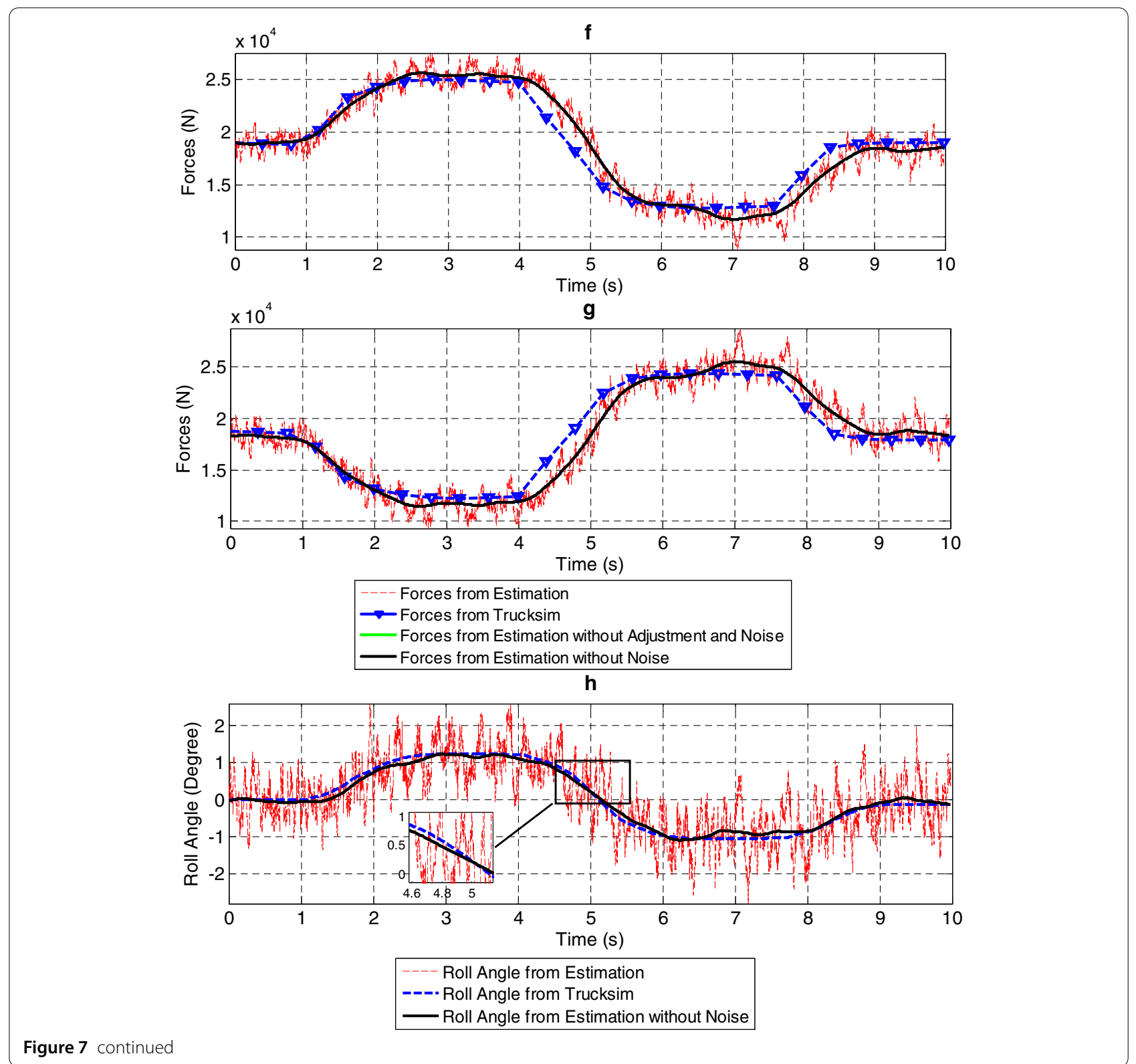

\section{Results and Discussion}

For validation of the proposed estimation method based on the ATEKF, the estimation is jointly simulated using MATLAB/Simulink and Trucksim software, which can simulate a real truck used in the heavy vehicle industry. To make the simulation closer to reality, noise is added to the data from Trucksim based on real experiments. The heavy truck in Figure 5 is tested, and the noises are acquired and inserted into the simulation data, the details of which are shown in Figure 5.

A step steer input is designed in Trucksim, as shown in Figure 6, which is used in the validation. Three different conditions (Conditions 1, 2, 3) are designed with different driver operations and loads (shown in Table 3), and the comparison results are shown in Figures 7, 8, and 9. The normalized errors (calculated as Eq. (36)) of these data in the figures are displayed in Table 4. This comparison demonstrates the accuracy of the proposed estimation system and the importance of an adjustment.

$$
\varepsilon=\frac{\left(\sum_{i=1}^{n}\left|z_{\text {estimated }}-z_{\text {measured }}\right|\right)}{n \max \left|\left(\max \left(z_{\text {measured }}\right)-\min \left(z_{\text {measured }}\right)\right)\right|} \times 100 .
$$



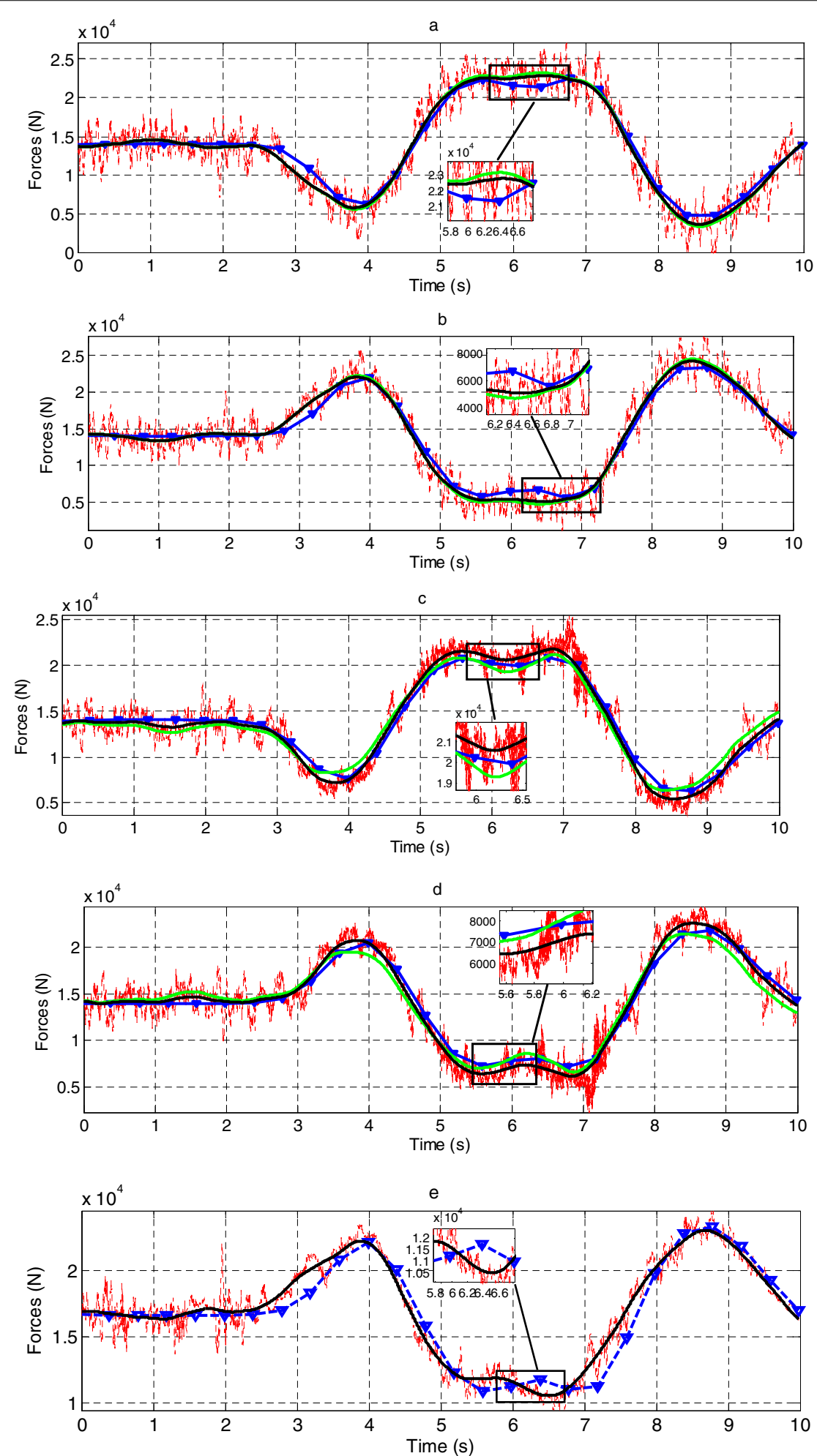

Figure 8 Comparisons between Trucksim and estimation under Condition 2 

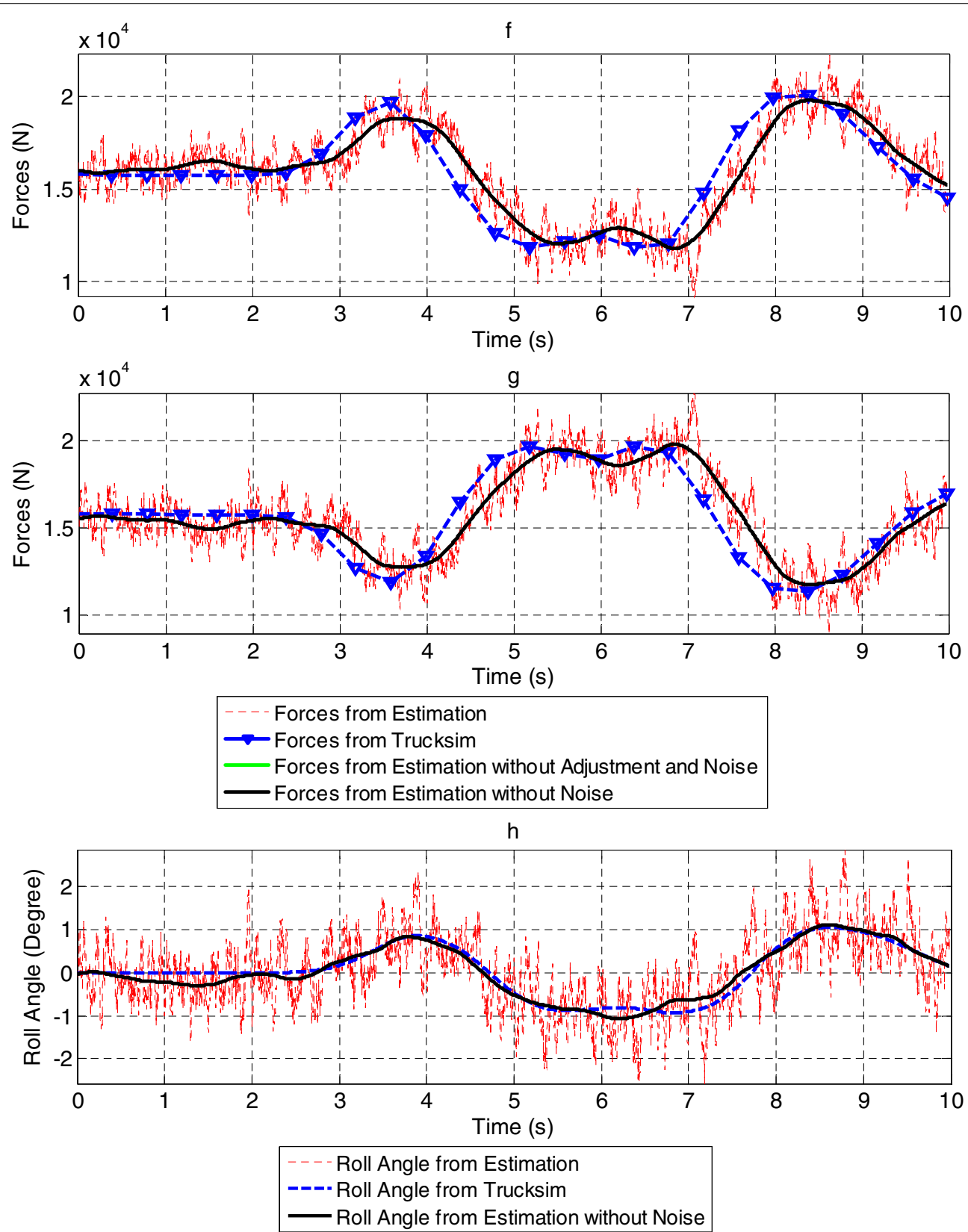

Figure 8 continued

In Eq. (36), $z_{\text {estimated }}$ is the variable from the estimation, $z_{\text {measured }}$ is the variable from Trucksim, and $n$ is the number of sampling points. This normalized error describes the percentage of error relative to the amplitude. An examination of the data in Table 4 shows that the normal errors of the ATEKF are globally less than 8\%; in addition, compared to the estimation system without the vertical tire force adjustment subsystem, the estimation with an adjustment can improve the accuracy by as much as 61\% (details are listed in "Appendix", Section 2).

In Figures 7, 8 and 9, the estimation system with and without an adjustment subsystem is compared. It can be clearly seen that the subsystem can adjust the vertical tire forces to be closer to the real values.

In Figures 7, 8 and 9, (a) shows the vertical tire forces of the fourth left tire, (b) indicates the forces of the fourth 

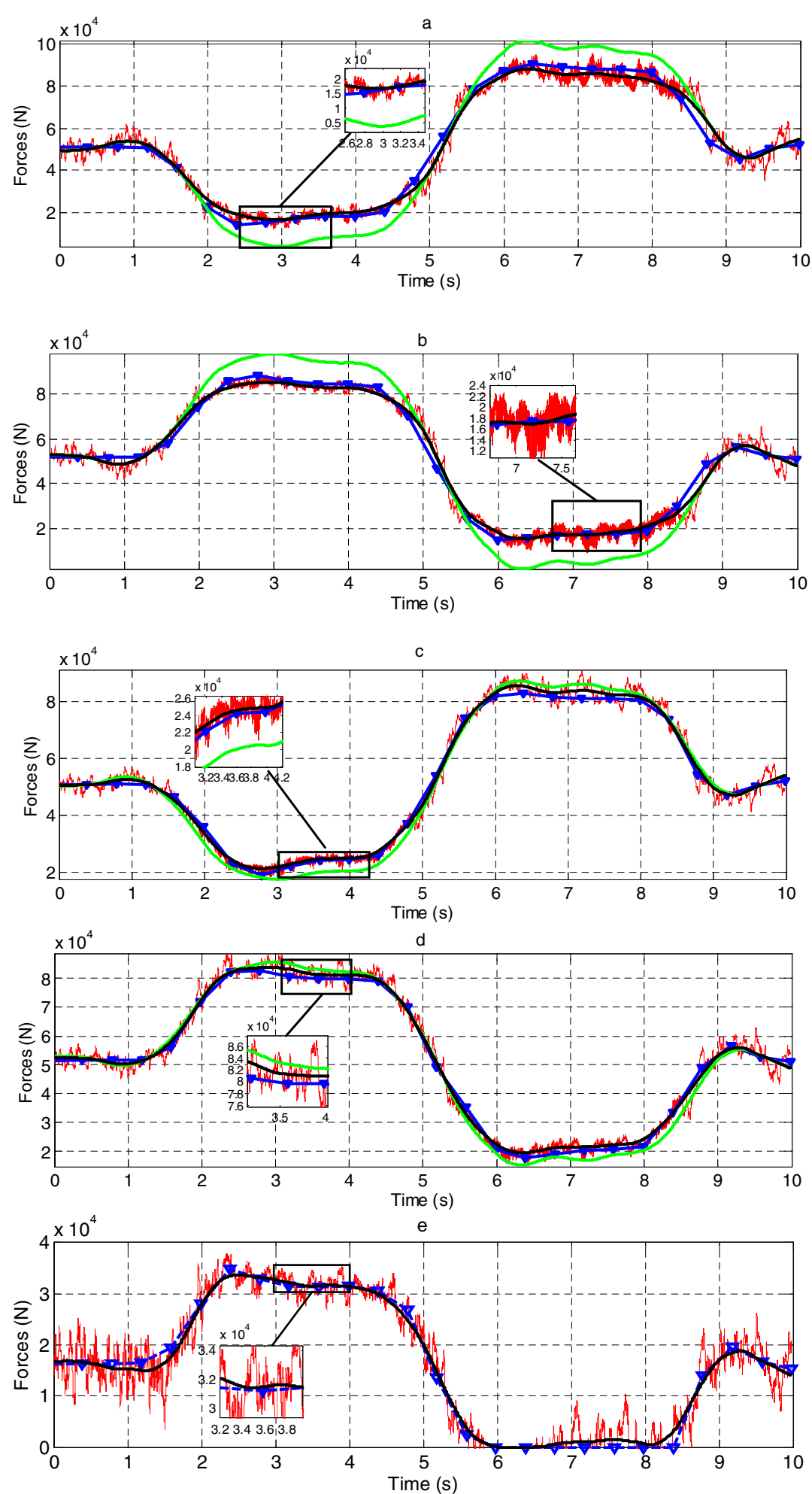

Figure 9 Comparisons between Trucksim and estimation under Condition 3 

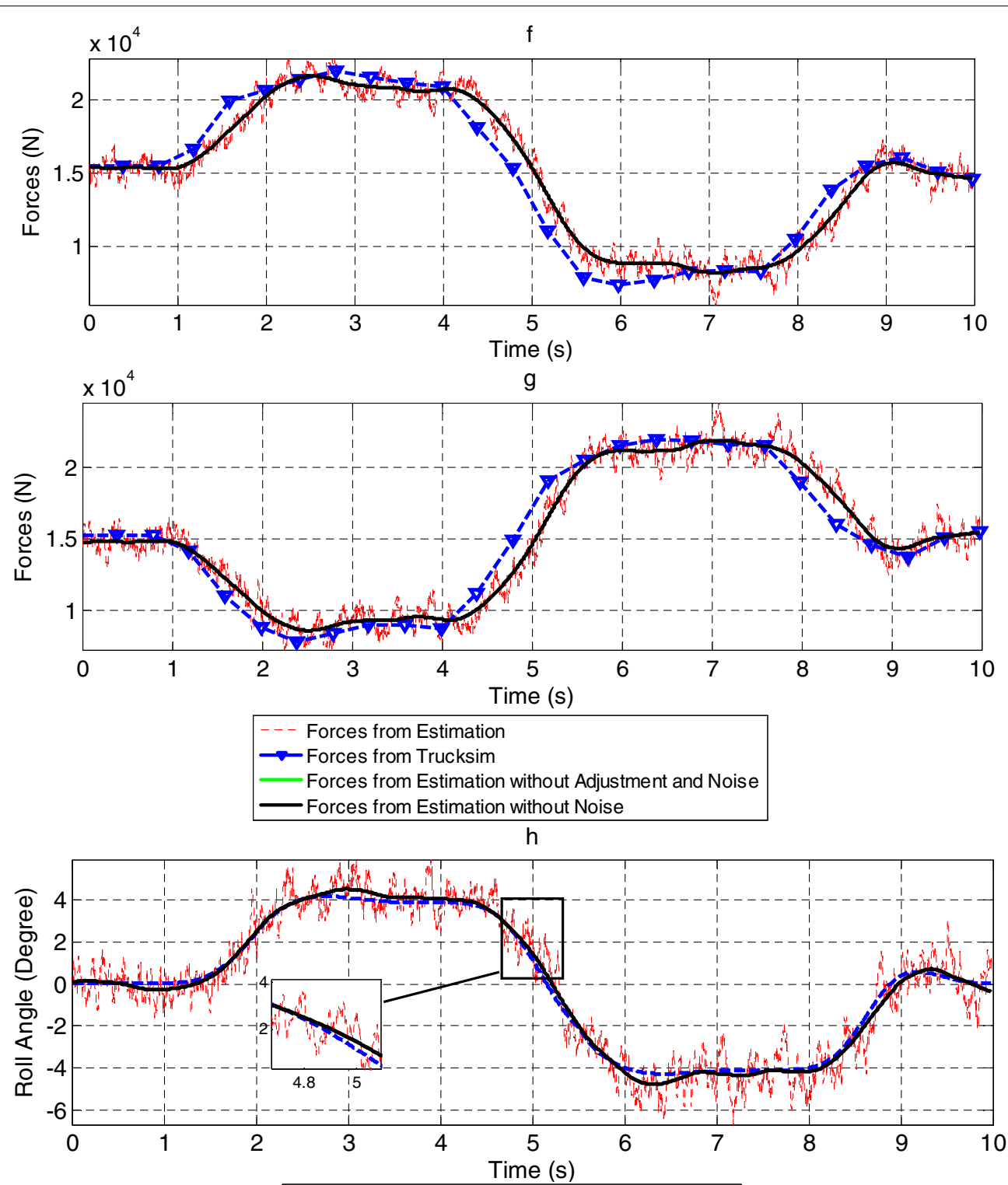

- - - Roll Angle from Estimation

---- Roll Angle from Trucksim

- Roll Angle from Estimation without Noise

Figure 9 continued

Table 4 Normalized errors (data without noise) (\%)

\begin{tabular}{|c|c|c|c|c|c|c|c|c|}
\hline & $F_{z r 1}$ & $F_{z r 2}$ & $F_{z r 3}$ & $F_{z r 4}$ & $F_{z / 1}$ & $F_{z / 3}$ & $F_{z / 4}$ & $\phi$ \\
\hline Condition 1 & 7.0244 & 1.7783 & 2.8073 & 2.3357 & 7.6067 & 2.7132 & 2.5681 & 2.9157 \\
\hline Condition 1 without adjustment & & & 6.3018 & 6.0018 & - & 6.0375 & 6.4347 & - \\
\hline Condition 2 & 5.9661 & 2.1749 & 3.5664 & 3.6854 & 5.7985 & 3.3871 & 4.0808 & 5.2541 \\
\hline Condition 2 without adjustment & & & 4.0826 & 3.9457 & - & 3.8438 & 4.3435 & - \\
\hline Condition 3 & 4.2111 & 5.8477 & 3.2823 & 3.5756 & 3.9356 & 3.1678 & 4.0651 & 2.2039 \\
\hline Condition 3 without adjustment & & & 4.7263 & 8.7285 & - & 5.1984 & 8.1338 & - \\
\hline
\end{tabular}




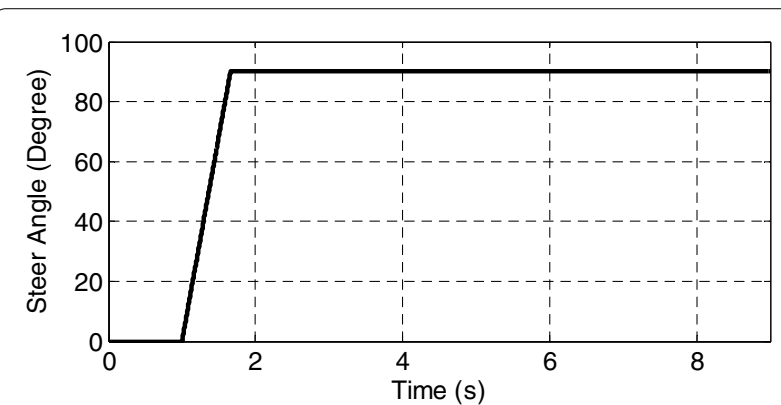

Figure 10 Steer angle of Condition 4

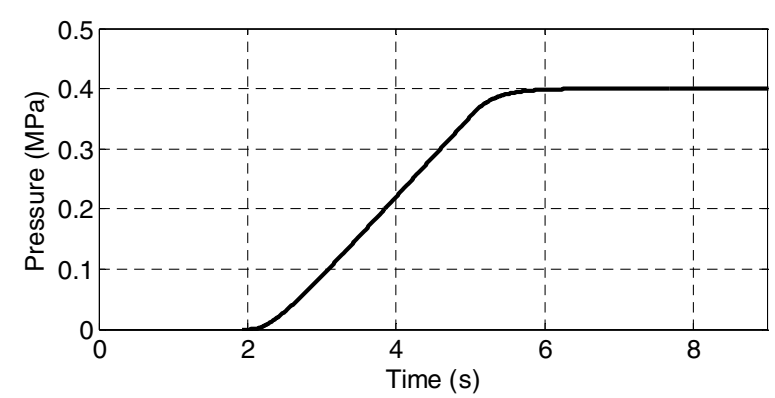

Figure 11 Pressure of air brake chamber under Condition 4

right tire, (c) shows the forces of the third left tire, (d) represents the forces of the third right tire, (e) shows the forces of the second right tire, (f) indicates the forces of the first right tire, $(\mathrm{g})$ shows the forces of the first left tire, and (h) represents the roll angle comparison. In the figures, the data without noise are the estimation system results after the filter is used.

To validate whether the estimation method can adapt to the load transfer under the combined conditions, a step steer operation with braking is designed as Condition 4 listed in Table 3. The steering angle and braking pressure are shown in Figures 10 and 11, and the road friction coefficient is 0.3 . Figure 12 shows the tire vertical forces from estimation under Condition 4. The estimation has a certain ability to deal with the combined condition.

Figure 13 shows the different performances of the ATEKF and TEKF, clearly demonstrating the advantages of the proposed ATEKF, and that the initial settings are not well modulated. Even without the initial parameter updates, the convergence of the ATEKF is better than that of the TEKF. Using both the initial settings update and the vertical force adjustments, the ATEKF can estimate the vertical tire forces with a satisfactory level of accuracy.

\section{Conclusions}

In this study, an estimation system was designed for the vertical tire forces of a four-axle truck used in vehicle dynamic control based on the proposed ATEKF. The model of a multi-axle vehicle with vertical tire forces was improved as the basis of the estimation. The truck body was separated into several parts to solve the complex overconstraint problem, and the different location, mass, and volume of the goods were also taken into consideration.
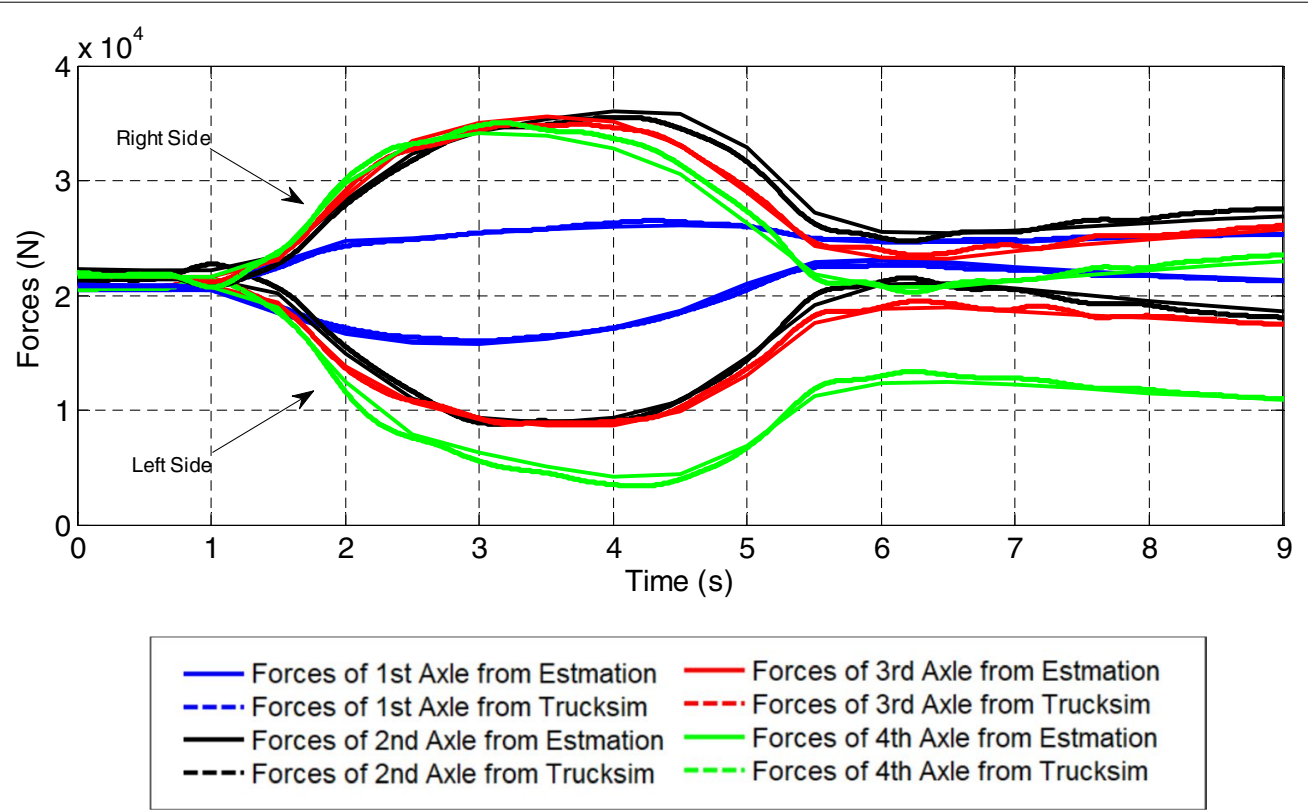

Figure 12 Vertical tire forces from estimation with adjustment under Condition 4 without noise 


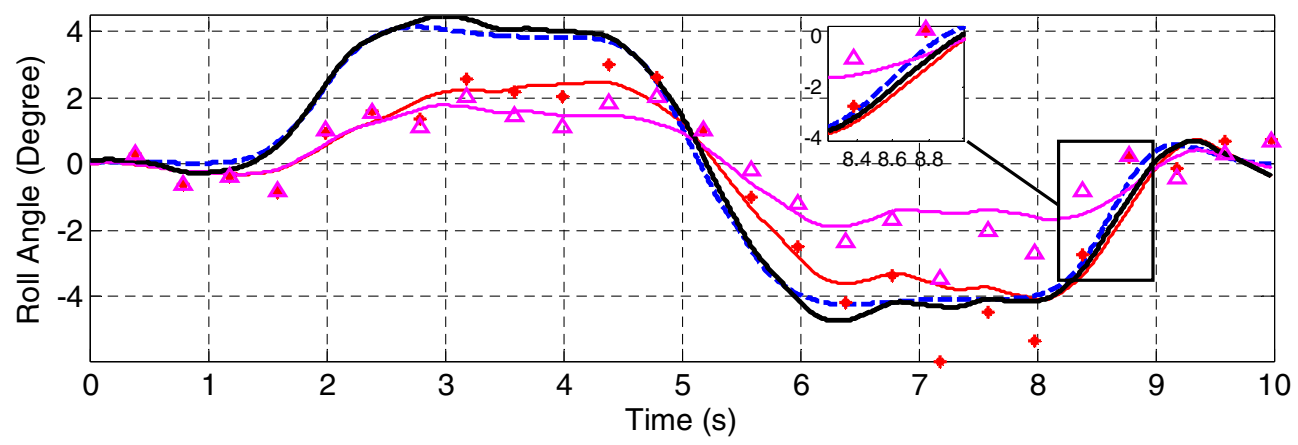

$$
\begin{aligned}
& - \text { Roll Angle from Estimation without Initial Parameters Update and Noise } \\
& - \text { Roll Angle from Trucksim } \\
& \text { Roll Angle from Estimation without Initial Parameters Update, Sliding Mode Update and Noise (TEKF) } \\
& \text { Roll Angle from Estimation without Noise (ATEKF) } \\
& \text { Roll Angle from Estimation without Initial Parameters Update } \\
& \text { Roll Angle from Estimation without Initial Parameters Update, Sliding Mode Update (TEKF) }
\end{aligned}
$$

Figure 13 Comparisons between ATEKF and TEKF under Condition 3

The proposed ATEKF had three EKFs for estimating the parameters and the states. The initial settings of the ATEKF were updated using a rough calculation method based on the changes to the cargo. The initial parameters used in the ATEKF estimation system can be measured when the truck is unloaded or static, and the location, height, and weight of the cargo loads can also be easily known. To make the simulation closer to reality, noises from actual experiments were added to the inputs of the estimation. During the estimation process, a sliding mode update was designed and added to the roll dynamic parameter update, which transforms the TEKF into the ATEKF. Compared with the TEKF, the ATEKF can achieve greater accuracy without precise measurements. The sensors used in this research are all commonly applied. The results showed that the proposed ATEKF algorithm can provide an excellent estimation of the states and parameters. For different cargo weights, heights, and different good locations, the ATEKF algorithm can provide accurate roll angles and usable parameters for the vertical tire force calculation. The force values are sufficiently accurate for adoption in dynamic vehicle control.

In future studies, the estimation system will be improved for determination of multi-axle truck parameters for both inhomogeneous and fluidic goods. More extreme operational conditions will also be considered. The bank and slope angles of the road will also be considered. Moreover, the estimation system will be mounted on a real truck. Finally, the parameters of Part 1 will also be regarded as unknown.

\section{Appendix}

The initial vertical tire forces calculation method:

$$
\begin{aligned}
F_{z 10}= & \frac{m_{c} g\left(\frac{\left(l_{3}-l_{2}\right)}{2}+\left(l_{2}-L_{c}\right)\right)}{\left(\frac{\left(l_{3}-l_{2}\right)}{2}+\left(l_{2}-\frac{l_{1}}{2}\right)\right)} \\
& +\frac{m_{\nu} g\left(\frac{\left(l_{v 30}-l_{\nu 20}\right)}{2}+l_{v 20}\right)}{\left(\frac{\left(l_{v 30}-l_{v 20}\right)}{2}+\left(l_{v 20}+l_{\nu 10}-\frac{\left(l_{v 0}-l_{v 10}\right)}{2}\right)\right)},
\end{aligned}
$$

$$
\left\{\begin{array}{l}
F_{z l 10, z r 10}=K_{s t r 12} F_{z 10}+\frac{m_{a 1} g}{2}, \\
F_{z l 20, z r 20}=K_{s t r 12} F_{z 10}+\frac{m_{a 2} g}{2} \\
F_{z l 30, z r 30}=K_{s t r 34}\left(\left(m_{v}+m_{c}\right) g-F_{z 10}\right)+\frac{m_{a 3} g}{2} \\
F_{z l 40, z r 40}=K_{s t r 34}\left(\left(m_{v}+m_{c}\right) g-F_{z 10}\right)+\frac{m_{a 4} g}{2},
\end{array}\right.
$$

$l_{\nu 0}=2.813 \mathrm{~m}, \quad l_{\nu 10}=l_{1}-l_{\nu 0}, \quad l_{v 20}=l_{2}-l_{\nu 0}$, $l_{v 30}=l_{3}-l_{\nu 0}$.

In the above equations, $l_{\nu 0}$ is the equivalent C.G position to the first axle, $K_{s t r 12}$ is the static load transfer ratio of the first and second axles, $K_{s t r 34}$ is for the third and fourth axles $\left(K_{\text {str12 }}=K_{\text {str } 34}=0.5\right)$.

The improvement percentage of adjustment calculation method:

$$
P=\max \left(\frac{(B-A)}{B}\right) .
$$

$A$ is the normalized errors of the forces with adjustment, $B$ is the normalized errors of the forces without adjustment, $P$ is the improvement percentage of adjustment calculation method. 


\section{Nomenclature}

\begin{tabular}{|c|c|c|}
\hline Symbol & Explanation & Details \\
\hline$g$ & $\begin{array}{l}\text { The acceleration of } \\
\text { gravity }\end{array}$ & $9.8 \mathrm{~m} / \mathrm{s}^{2}$ \\
\hline$m$ & $\begin{array}{l}\text { Total mass of the } \\
\text { vehicle }\end{array}$ & $m=m_{V}+\sum_{i=1}^{4} m_{a i}+m_{C}$ \\
\hline$m_{s}$ & Mass of sprung mass & $m_{s}=m_{v}+m_{c}$ \\
\hline$m_{a i}$ & Mass of axle & $\begin{array}{l}m_{a 1}=570 \mathrm{~kg} \\
m_{a 2}=m_{a 3}=m_{a 4}=760 \mathrm{~kg} \\
i=1,2,3,4\end{array}$ \\
\hline$m_{v}$ & Mass of vehicle body & $4457 \mathrm{~kg}$ \\
\hline$m_{c}$ & Mass of cargo load & \\
\hline$m_{i}$ & $\begin{array}{l}\text { The cargo mass on } \\
\text { Part } i\end{array}$ & $i=1,2,3$ \\
\hline$v_{x}$ & Vehicle velocity & \\
\hline$\varphi$ & Roll angle & \\
\hline$a_{x}, a_{y}$ & $\begin{array}{l}\text { Longitudinal and } \\
\text { lateral acceleration }\end{array}$ & \\
\hline$F_{z l i_{0}, z r_{0}}$ & $\begin{array}{l}\text { Initial vertical tire } \\
\text { forces }\end{array}$ & $i=1,2,3,4$ \\
\hline$F_{z r i, z l i}$ & Vertical forces of tires & $i=1,2,3,4$ \\
\hline$K_{b i}$ & $\begin{array}{l}\text { Stiffness of anti-roll } \\
\text { bar }\end{array}$ & $\begin{array}{l}K_{b 1}=73020 \mathrm{Nm} / \mathrm{rad} \\
K_{b 3, b 4}=K_{b 2}\end{array}$ \\
\hline$K_{i}$ & $\begin{array}{l}\text { Suspension stiffness } \\
\text { of parts }\end{array}$ & $K_{i}=250000 \mathrm{Nm} / \mathrm{rad}, i=1,2,3$ \\
\hline$C_{i}$ & $\begin{array}{l}\text { Suspension dampness } \\
\text { of parts } i\end{array}$ & $C_{i}=33000 \mathrm{Nms} / \mathrm{rad}, i=1,2,3$ \\
\hline K & $\begin{array}{l}\text { Suspension stiffness } \\
\text { of whole truck }\end{array}$ & \\
\hline C & $\begin{array}{l}\text { Suspension dampness } \\
\text { of whole truck }\end{array}$ & \\
\hline H & Wheel track & $2.03 \mathrm{~m}$ \\
\hline$H_{c}$ & Width of the cargo & $1.75 \mathrm{~m}$ \\
\hline$I_{v 1}$ & $\begin{array}{l}\text { Distance between } \\
\text { unloaded c.g1 point } \\
\text { and front axle }\end{array}$ & $1.113 \mathrm{~m}$ \\
\hline$l_{i}$ & $\begin{array}{l}\text { Distance between first } \\
\text { axle and the ith axle }\end{array}$ & $\begin{array}{l}l_{1}=4.194 \mathrm{~m}, l_{2}=6 \mathrm{~m} \\
l_{3}=7.806 \mathrm{~m}, i=2,3,4\end{array}$ \\
\hline$I_{r 1 i}$ & $\begin{array}{l}\text { Distance between } \\
\text { c.g1 point and }(i-1) \\
\text { th axle }\end{array}$ & $I_{r 1 i}=I_{i}-I_{v 1,} i=1,2,3$ \\
\hline$d$ & Height of cargo & \\
\hline$h_{b}$ & $\begin{array}{l}\text { Distance between } \\
\text { cargo floor to } \\
\text { ground }\end{array}$ & $1.25 \mathrm{~m}$ \\
\hline$h_{d}$ & $\begin{array}{l}\text { Distance between a } \\
\text { hypothesis roll axle } \\
\text { and vehicle roll axle }\end{array}$ & $1.073 \mathrm{~m}$ \\
\hline$h$ & $\begin{array}{l}\text { The distance between } \\
\text { C.G to roll axle }\end{array}$ & \\
\hline$h_{r i}$ & $\begin{array}{l}\text { The distance between } \\
\text { c.gi to roll axle of } \\
\text { different parts }\end{array}$ & $\begin{array}{l}h_{r 1}=0.5 \mathrm{~m}, h_{r 2}=h_{r 3}, i=1 \\
\quad 2,3\end{array}$ \\
\hline$h_{i}$ & $\begin{array}{l}\text { The c.gi height of dif- } \\
\text { ferent parts }\end{array}$ & $h_{1}=1.173 \mathrm{~m}, i=1,2,3$ \\
\hline
\end{tabular}

\begin{tabular}{|c|c|c|}
\hline Symbol & Explanation & Details \\
\hline$L_{K}$ & Length of the cargo & \\
\hline$L_{c}$ & $\begin{array}{l}\text { The centre of the } \\
\text { cargo mass to the } \\
\text { first axle }\end{array}$ & \\
\hline$L_{i}$ & $\begin{array}{l}\text { Length of the goods } \\
\text { on the Part } i\end{array}$ & $i=1,2,3$ \\
\hline$J_{x}$ & $\begin{array}{l}\text { Rotational inertia of } \\
\text { truck to roll axle }\end{array}$ & \\
\hline$J_{x c}$ & $\begin{array}{l}\text { Rotational inertia of } \\
\text { cargo about } X \text { axis }\end{array}$ & \\
\hline$J_{x t}$ & $\begin{array}{l}\text { Unloaded truck rota- } \\
\text { tional inertia about } \\
\text { X axis }\end{array}$ & \\
\hline$I_{s 1}$ & $\begin{array}{l}\text { Distance between } \\
\text { the first separation } \\
\text { point to the first axle }\end{array}$ & \\
\hline$I_{c i}$ & $\begin{array}{l}\text { Distances between } \\
c . g_{1}, c . g_{2} \text { and } c . g_{3} \text { to } \\
\text { the second axle and } \\
\text { the third axle }\end{array}$ & $i=1,2,3$ \\
\hline$K_{J \times 1}$ & Gain for the $a_{0}$ & $K_{J x 1}=2 \frac{m_{c}}{5000}$ \\
\hline$K_{J \times 2}$ & Gain for the $a_{0}$ & $8 \times 10^{-6}$ \\
\hline$K_{1 \text { may }}$ & Gain of Rule 3 axle 3 & 37000 \\
\hline$K_{2 m a y}$ & Gain of Rule 3 axle 4 & 11000 \\
\hline$K_{3 \text { may }}$ & Gain of Rule 3 axle 4 & 70000 \\
\hline$K_{a}$ & Gain of $a$ & 100000 \\
\hline$k_{/ t r 3, / t r 4}$ & $\begin{array}{l}\text { Factors of rear magni- } \\
\text { fication }\end{array}$ & $k_{l t r 3, \operatorname{lt} 4}=1.5$ \\
\hline$T$ & Step size & 0.001 \\
\hline$R^{\tau}$ & Noise covariance & {$\left[\begin{array}{cc}0.0001 & 0 \\
0 & 0.0001\end{array}\right]$} \\
\hline$R^{e}$ & Noise covariance & $8.9 \times 10^{-4}$ \\
\hline$R^{n}$ & Noise covariance & 20 \\
\hline$R^{f}$ & Noise covariance & $1.06 \times 10^{7}$ \\
\hline$x_{0}$ & Initial value & {$\left[\begin{array}{ll}0 & 0\end{array}\right]^{\top}$} \\
\hline$v_{0}$ & Initial value & {$\left[\begin{array}{llll}a_{0} & 68.1565 & 10.9603\end{array}\right]^{\top}$} \\
\hline$w_{0}$ & Initial value & {$\left[\frac{d}{2}+h_{b} 1.71\right]^{\top}$} \\
\hline$P_{x_{0}}$ & Initial value & {$\left[\begin{array}{cc}0.127 & -0.205 \\
-0.205 & 0.1816\end{array}\right] \times 10^{-3}$} \\
\hline$P_{v_{0}}$ & Initial value & $0.001 \times 13 \times 3$ \\
\hline$P_{w_{0}}$ & Initial value & $0.0001 \times 13 \times 3$ \\
\hline
\end{tabular}

\section{Acknowledgements}

Not applicable.

\section{Authors'Contributions}

BZ was in charge of the whole trial and built the model and algorithm, TX and HW wrote the manuscript, GC and YH assisted with sampling and laboratory analyses. All authors read and approved the final manuscript.

\section{Authors' Information}

Buyang Zhang, born in 1989, graduated from College of Automotive Engineering, Jilin University, China. He is currently a researcher in Jihua Laboratory. His research interests include vehicle dynamic, vehicle stability control, intelligent tire, non-pneumatic tire. 
Ting Xu, born in 1990, graduated from College of Automotive Engineering, Jilin University, China. Her research interests include vehicle stability control, intelligent tire, non-pneumatic tire, tire modeling. She is currently a researcher in Jihua Laboratory.

Hong Wang, born in 1986, is currently a researcher in Tsinghua Intelligent Vehicle Design and Safety Research Institute, Tsinghua University. Her research interests include vehicle stability control, intelligent vehicle and new energy vehicle.

Yanjun Huang, born in 1986, is currently a post doctor at University of Waterloo, Canada. His research interests include vehicle stability control, intelligent vehicle and new energy vehicle.

Guoying Chen, born in 1984, is currently a master candidate at State Key Laboratory of Automotive Simulation and Control, Jilin University, China.

\section{Funding}

Supported by Basic and Applied Basic Research Foundation of Guangdong Province of China (Grant No. 2019A1515110763).

\section{Competing Interests}

The authors declare no competing financial interests.

\section{Author Details}

${ }^{1}$ Jihua Laboratory, Foshan 528200, Guangdong, China. ${ }^{2}$ State Key Laboratory of Automotive Simulation and Control, Jilin University, Changchun 130022, China. ${ }^{3}$ Tsinghua Intelligent Vehicle Design and Safety Research Institute, Tsinghua University, Beijing 100084, China. ${ }^{4}$ Department of Mechanical and Mechatronics Engineering, University of Waterloo, Waterloo, Canada.

Received: 15 February 2020 Revised: 8 March 2021 Accepted: 23 April 2021

Published online: 05 June 2021

\section{References}

[1] M Doumiati, A Victorino, A Charara, et al. Lateral load transfer and normal forces estimation for vehicle safety: Experimental test. Vehicle System Dynamics, 2009, 47(12): 1511-1533.

[2] GP Daniel, Y Jorge, O Oluremi, et al. A strain-based method to estimate slip angle and tire working conditions for intelligent tires using fuzzy logic. Sensors, 2017, 17(4): 874

[3] C Francesco, P Gianluca, R Nicola, et al. A multi sensing setup for the intelligent tire monitoring. Sensors, 2017, 17(3): 576.

[4] H Lee, STaheri. Intelligent tires - A review of tire characterization literature. IEEE Intelligent Transportation Systems Magazine, 2017, 9(2): 114-135.

[5] R Ghandour, A C Victorino, M Doumiati, et al. Tire/road friction coefficient estimation applied to road safety. 18th Mediterranean Conference on Control and Automation, 2010, 20: 1485-1490.

[6] M Doumiati, A C Victorino, A Charara, et al. Onboard real-time estimation of vehicle lateral tire-road forces and sideslip angle. IEEE/ASME Transactions on Mechatronics, 2011, 16(4): 601-614

[7] M Doumiati, A Victorino, D Lechner, et al. Observers for vehicle tyre/ road forces estimation: experimental validation. Vehicle System Dynamics, 2010, 48(11): 1345-1378

[8] H P Wang, GIY Mustafa, YTian. Model-free fractional-order sliding mode control for an active vehicle suspension system. Advances in Engineering Software, 2017, 115

[9] Y Mohammadi, S Ganjefar. Quarter car active suspension system: minimum time controller design using singular perturbation method. International Journal of Control Automation \& Systems, 2017, 15(1): 1-13.

[10] P Xiao, H Gao, L Niu. Research on magnetorheological damper suspension with permanent magnet and magnetic valve based on developed
FOA-optimal control algorithm. Journal of Mechanical Science \& Technology, 2017, 31(7): 3109-3119.

[11] S Khaleghian, A Emami, S Taheri. A technical survey on tire-road friction estimation. Friction, 2017, 5(2): 123-146.

[12] M Wielitzka, M Dagen, T Ortmaier. State estimation of vehicle's lateral dynamics using unscented Kalman filter. Decision and Control, 2015: 5015-5020.

[13] M Doumiati, A Victorino, A Charara, et al. Virtual sensors, application to vehicle tire-road normal forces for road safety. American Control Conference, 2009: 3337-3343.

[14] Z Ma, Y Zhang, J Yang. Velocity and normal tyre force estimation for heavy trucks based on vehicle dynamic simulation considering the road slope angle. Vehicle System Dynamics, 2015, 54(2): 137-167.

[15] W Cho, J Yoon, S Yim, et al. Estimation of tire forces for application to vehicle stability control. IEEE Transactions on Vehicular Technology, 2010, 59(2): 638-649.

[16] X Ying, B Deng, X Gang. Estimation of vehicle states and road friction based on DEKF. International Conference on Power Electronics Systems and Applications, 2016: 1-7.

[17] M Aldimirov, R Arnaudov. Method for automated reconstruction of a car's path during crash from GPS/INS data using a Kalman filter. Advances in Engineering Software, 2017.

[18] B C Chen, F C Hsieh. Sideslip angle estimation using extended kalman filter. Vehicle System Dynamics, 2008, 46(sup1): 353-364.

[19] I Kim, J Bang, K Huh. Estimation of the climbing angle in the presence of yawing motion. Proceedings of the Institution of Mechanical Engineers Part D Journal of Automobile Engineering, 2015, 229(9): 1263-1275.

[20] G R Jiang, X L Miao, Y H Wang, et al. Real-time estimation of the pressure in the wheel cylinder with a hydraulic control unit in the vehicle braking control system based on the extended Kalman filter. Proceedings of the Institution of Mechanical Engineers Part D Journal of Automobile Engineering, 2017, 231(10): 1340-1352.

[21] G R Jiang, L F Liu, C H Guo, et al. A novel fusion algorithm for estimation of the side-slip angle and the roll angle of a vehicle with optimized key parameters. Proceedings of the Institution of Mechanical Engineers Part D Journal of Automobile Engineering, 2017, 231(2): 161-174.

[22] M Haudum, J Edelmann, M Plöchl, et al. Vehicle side-slip angle estimation on a banked and low-friction road. Proceedings of the Institution of Mechanical Engineers Part D Journal of Automobile Engineering, 2018, 232(12): 1584-1596.

[23] AH Ahangarnejad, S Baslamisli. Adap-tyre: DEKF filtering for vehicle state estimation based on tyre parameter adaptation. International Journal of Vehicle Design, 2016, 71(1/2/3/4): 52.

[24] C F Zong, D Hu, HY Zheng. Dual extended kalman filter for combined estimation of vehicle state and road friction. Chinese Journal of Mechanical Engineering, 2013, 26(2): 313-324.

[25] T A Wenzel, K J Burnham, M V Blundell, et al. Dual extended kalman filter for vehicle state and parameter estimation. Vehicle System Dynamics, 2006, 44(2): 153-171.

[26] B Y Zhang, C F Zong, G Y Chen, et al. A novel integrated stability control based on differential braking and active steering for four-axle trucks. Chinese Journal of Mechanical Engineering, 2019, 32:12, https://doi. org/https://doi.org/10.1186/s10033-019-0323-0.

[27] J O Hahn, R Rajamani, L Alexander. Gps-based real-time identification of tire-road friction coefficient. IEEE Transactions on Control Systems Technology, 2002, 10(3): 331-343.

[28] C Larish, D Piyabongkarn, V Tsourapas, et al. A new predictive lateral load transfer ratio for rollover prevention systems. IEEE Transactions on Vehicular Technology, 2013, 62(7): 2928-2936.

[29] T J Zhu, C F Zong. Rollover prevention for heavy trucks using robust control. 2009 Second ISECS International Colloquium on Computing, Communication, Control, and Management, 2009, 3: 182-185. 